\title{
Anti-Inflammatory Effects of Chinese Herbal Medicine on COPD: A Systematic Review
}

Miao $\mathbf{Q}^{1}$, Cong $\mathrm{X}^{1}$, Du $\mathrm{Y}^{2}$, Wang $\mathrm{B}^{1}$, Qiao $\mathrm{CY}^{3}$ and An $\mathrm{X}^{4 *}$

${ }^{1}$ No 1 Caochang St, Haidian district, Beijing, 100091, China

${ }^{2}$ Midwestern University 555 31st Street Downers Grove, IL, 60515, USA

${ }^{3}$ School of Health Sciences and Business, Monash University, Australia

${ }^{4} \mathrm{H}$ and $\mathrm{J}$ CRO PTY. LTD, Australia

\begin{abstract}
Background: Airway inflammation and inflammatory mediators play an imperative role in the pathogenesis of COPD. Currently, understanding of the anti-inflammatory effect of Chinese herbal medicine (CHM) on COPD is limited, and CHM's mechanism of actions is unclear. This systematic review (SR) evaluates anti-inflammatory effects of $\mathrm{CHM}$ on the concentration of various inflammatory mediators, such as Tumor Necrosis Factor-alpha (TNF- $\alpha$ ) and interleukin-8 (IL-8), in the sputum and serum of COPD patients.
\end{abstract}

Methods: The studies chosen for this SR were obtained from Chinese and English databases. The study selection criteria were based on randomized, controlled trials of stable COPD patients on adjunct oral CHM; and the changes in concentration of inflammatory mediators post-treatment were analyzed via meta-analysis.

Results: 2,268 patients in 29 studies were evaluated. 2 studies were assessed to be of low-risk in all domains. The results showed significant reduction in the serum level of IL-8 (mean: -1.27 and $95 \%$ confidence interval $(\mathrm{Cl}$ ) $[-1.86,-0.68]$ ) and TNF- $\alpha$ (Mean: -0.72 and $95 \% \mathrm{Cl}[-1.01,-0.43])$ in patients treated with CHM plus bronchodilators, compared to bronchodilators alone.

Conclusion: This SR explains CHM's mechanism of action, and demonstrates CHM's anti-inflammatory effects on patients with stable COPD.

Keywords: Chinese herbal medicine; Chronic obstructive pulmonary disease; Tumor necrosis factor- $\alpha$; Interleukin-8; Systematic review

\begin{abstract}
Abbreviations AE: Adverse Events; BALF: Bronchial Alveolar Lavage Fluid; CENTRA: Cochrane Central Register of Controlled Trials; CHM: Chinese herbal medicine; CI: Confidential Interval; CINAHL: Cumulative Index to Nursing and Allied Health Literature; CNKI: China National Knowledge Infrastructure; COPD: Chronic obstructive pulmonary disease; CONSORT: Consolidated Standards of Reporting Trials; CQVIP: Chongqing VIP; GRADE: Grading of Recommendations Assessment, Development and Evaluation; IL6: Interleukin-6; IL-8: Interleukin-8; MD: Mean Difference; MMP9: Matrix Metalloproteinase-9; MOA: Mechanism Of Action; QoL: Quality of Life; PRISMA: Systematic Reviews and Meta-Analyses; RCTs: Randomized Controlled Trials; SR: Systematic Review; Std. MD: Standardized Mean Difference; TGF- $\beta 1$ : Transforming Growth Factor- $\beta 1$; TNF- $\alpha$ : Tumor Necrosis Factor-alpha
\end{abstract}

\section{Introduction}

Chronic obstructive pulmonary disease (COPD) is a complex disease with multiple pathogeneses [1]. Airway inflammation plays an imperative role in the pathogenesis of COPD. A wide range of inflammatory mediators are associated with COPD; interleukin- 8 (IL-8), IL-6, Tumor Necrosis Factor-alpha (TNF- $\alpha$ ), and matrix metalloproteinase (MMP-9) have been shown to induce neutrophil production, alveolar macrophages release, emphysema formation, and lung remodeling [2]. Compared to healthy subjects, patients with stable COPD had an increased expression of inflammatory mediators, particularly in sputum and serum [3,4]. In addition, these inflammatory mediators correlated with clinical outcomes of lung function, BODE index, frequency of COPD exacerbation, and severity and mortality of COPD [5-7].

The use of Chinese herbal medicine ( $\mathrm{CHM}$ ) as an adjunct therapy for COPD has been documented in more than one hundred clinical trials over the past decade. Previous systematic reviews (SR) have shown that oral CHM provided symptom relief, improved Quality of life (QoL) and lung function, and reduced frequency of COPD exacerbation $[8,9]$. However, understanding of CHM's anti-inflammatory effects is limited and CHM's mechanism of action (MOA) is not clear. This study aims to investigate the effects of CHM on inflammatory mediators in induced sputum and serum in patients with stable COPD, as well as its MOA.

\section{Materials and Method}

This SR was conducted by Standard for Systematic Review [10] and guided by Preferred Reporting Items for Systematic Reviews and MetaAnalyses statement (PRISMA) [11].

Search strategy included identifying search databases and search terms. Relevant studies were selected from both English and Chinese databases. English databases included PubMed, CINAHL, and CENTRAL (Cochrane Central Register of Controlled Trials); Chinese databases included CNKI, CQVIP and Wan fang. Appropriate search terms (per guideline of Cochrane Airways Group) were used to identify appropriate studies. Potential studies were chosen from their respective inceptions until August 2014, without language restrictions.

Search terms were identified through PubMed using medical subject headings $(\mathrm{MeSH})$ relevant to COPD and from the Cochrane Airways Group Specialized Register of COPD trials. These terms were separated into those relevant to COPD, such as 'Pulmonary disease,

*Corresponding author: $\mathrm{An} \mathrm{X}, \mathrm{H}$ and J CRO PTY. LTD, Australia, Tel: 0061401777369; E-mail: xuedongan@hotmail.com

Received February 25, 2016; Accepted May 10, 2016; Published May 16, 2016

Citation: Miao Q, Cong X, Du Y, Wang B, Qiao CY, et al. (2016) Anti-Inflammatory Effects of Chinese Herbal Medicine on COPD: A Systematic Review. Lung Dis Treat 2: 107. doi:10.4172/2472-1018.1000107

Copyright: $\odot 2016$ Miao Q, et al. This is an open-access article distributed under the terms of the Creative Commons Attribution License, which permits unrestricted use, distribution, and reproduction in any medium, provided the original author and source are credited. 
chronic obstructive' etc.; relevant to randomized clinical trials such as 'Clinical trials', 'Randomized controlled trials', etc.; relevant to Chinese medicine such as 'Traditional Chinese Medicine' and 'Herbal Medicine'.

\section{Study selection criteria}

The criteria for study inclusion were based on study type, patient population, treatment method, and resulting outcomes. Studies that qualified for all previously stated criteria included: randomized controlled trials (RCTs) with a parallel group design; patients with stable COPD without complication of asthma, bronchiectasis, corpulmonale, or pulmonary hypertension; interventions that used oral administration of CHM; outcomes that focused on testing biomarkers of IL- 8 , TNF- $\alpha$, in serum and sputum, transforming growth factor- $\beta 1$ (TGF- $\beta 1$ ), and IL-6 in serum.

\section{Assessment of methodological quality}

The methodological quality of each study's risk-of-bias was assessed by Cochrane Collaboration, which consists of six domains: Sequence Generation, Allocation Concealment, Blinding Method and Outcomes Assessment, Incomplete Data, Selective Reporting and Other Bias [12]. The risk-of-bias of each study was evaluated as low, high or unclear. Selective Reporting was assessed by each study's reporting protocol. Other Bias was assessed by comparing baseline data and method of statistical analysis in each respective study.

\section{The quality of grading of recommendation}

Grading of Recommendations Assessment, Development and Evaluation (GRADE) and GRADEpro [13] were used; the quality rating of each study was evaluated as high, moderate, low, or very low. Assessment of quality-of-evidence was based on Risk-of-Bias, Inconsistency, Indirectness, Imprecision, and Publication Bias. The Large Effect, Plausible Confounding Variables, and Dose-Response Gradient were evaluated as strongly supportive, weakly supportive, strongly oppose, or weakly oppose.

\section{Outcome measure}

The outcomes were mainly focused on the level of IL-8 examined and TNF- $\alpha$ in serum and sputum, and also involved the level of transforming growth factor- $\beta 1$ (TGF- $\beta 1$ ), and IL-6 in serum.

\section{Data extraction and collection}

The relevance of title, abstract, and citations were assessed by two reviewers. Full articles were assessed by two reviewers. The Methodological Quality was assessed by two reviewers, audited by a third reviewer. The decision making process to include potential studies is outlined as a flow diagram based on the template provided by PRISMA [11]. Details of each study's treatment regimen were reported as RCTs using Consolidated Standards of Reporting Trials (CONSORT) [14].

\section{Data synthesis and analysis}

Datasets were analyzed with RewMan 5.3. The continuous data was expressed as Mean Difference (MD), standardized MD (Std. MD), and 95\% Confidential Interval (CI). The model of Random Effects was applied for heterogeneity. All data sets were imported from RewMan and assessed by GRADEpro.

\section{Results}

3,886 potential studies were initially identified. 3,143 remained after duplicates were eliminated. Further screening resulted in the exclusion of 2,921 studies for various reasons: 323 were not RCTs (case reports, surveys, retrospective studies, etc.), 333 did not relate to COPD or had additional complications of respiratory failure, heart failure, pulmonary hypertension or cor-pulmonale, 94 had inappropriate treatment regimen, 965 did not include biomarkers in its outcomes, 212 were non-human trials, 807 were review articles, and 187 used non-CHM adjunct therapy. Of the 222 remaining studies, 81 did not administer oral CHM, 66 did not include desired outcomes, and 3 had patients with non-stable COPD (such as COPD exacerbation or unidentified COPD courses) as well as 3 others. 29 studies met all required criteria, and were retrieved and analyzed in this SR (Figure 1).

\section{Demographic information}

Of the twenty nine studies, 27 were conducted in China and 2 in Japan. All of them were designed as parallel RCTs. Participants in each study were diagnosed with stable COPD, in accordance with the GOLD guideline modified by the CSRD [15]. Six studies [16-21] were done in out-patient settings only; one study [22] was in in-patient setting only, and five studies included participants in both out-patient and inpatient settings. Average age of participates ranged from $54.3 \pm 4.7$ to 72 in all studies except three, which did not specify age range [23-25]. 2,268 subjects were randomly selected and 2,193 subjects completed the entirety of their respective assessments, 75 subjects withdrew. 1,359 male subjects and 709 female subjects were identified, with the exception of two studies that did not include the gender of the participants $[24,26]$. Five studies identified the severity of COPD of their subjects as mild, moderate, or severe based on the GOLD guideline [19,27-30]. Seventeen studies defined COPD's Differentiation of Syndrome in Chinese Medicine (CM) to be: lung and spleen qi deficiency [19,20,31$33]$, lung and kidney qi deficiency $[21,22,27-29,34,35]$, or qi deficiency and blood stasis $[17,23,30,36,37]$; while other studies did not clearly define its differentiation of syndromes. Duration of treatment varied from: six months in seven studies [18,24,26,29,33-35], three months in nine studies [17,19-21,23,27,28,30,37], two months in six studies $[16,19,22,31,38,39]$, six weeks in one study [40], one month in five studies [25,36,41-43], and two weeks in one study [44] (Table 1).

\section{Intervention}

Medication regimens were based on GOLD guideline for management of patient with stable COPD [45]. Bronchodilators such as inhaled beta-2-agonists (salbutamol and salmeterol), anticholinergics (Tiotropium bromide), or theophylline tablets with salmeterol/ fluticasone propionate were used as mainstay treatments. Experimental groups consisted of CHM formulae or extraction of a single Chinese herb plus one drug of any category of bronchodilators. Control groups consisted of one drug of any category of bronchodilators alone or with placebo.

Oral forms of CHM formulae include oral liquid, capsules, granules, powder, or decoctions; their respective dosages are shown in Table 2. In seven studies $[19,27,29,33,35,41,43]$, the CHM formula were produced as granule or capsule by pharmaceutical companies certified with Good Manufacturing Practice with strict quality control. Each CHM formula is comprised of 1 to 16 herbs, from a total of 77 different kinds of herbs. The most commonly used herbs in all studies were Huang Qi (Astragalus membranaceus), Gan Cao (Glycyrrhiza uralensis), Chen Pi (Citrus reticulate), Dang Shen (Codonopsis pilosula), Di Huang (Rehmannia glutinosa) and Fu Ling (Poria cocos) (Table 2).

\section{Assessment of methodological quality}

Sequence Generation in thirteen studies [16-18,21,27,29-31,33,35$37,42]$ were low risk. Allocation Concealment in three studies $[27,31,40]$ 
Citation: Miao Q, Cong X, Du Y, Wang B, Qiao CY, et al. (2016) Anti-Inflammatory Effects of Chinese Herbal Medicine on COPD: A Systematic Review. Lung Dis Treat 2: 107. doi:10.4172/2472-1018.1000107

Page 3 of 11

\begin{tabular}{|c|c|c|c|c|c|c|c|c|}
\hline $\begin{array}{l}\text { First author, } \\
\text { Reference No. }\end{array}$ & Location & $\begin{array}{l}\text { Out /in } \\
\text { patients }\end{array}$ & $\begin{array}{l}\text { No. subjects } \\
\text { (R/A) }\end{array}$ & No. $M / F$ & $\begin{array}{c}\text { Age } \\
\text { Mean SD (years) }\end{array}$ & $\begin{array}{l}\text { *Severity of COPD/ } \\
\text { No. subjects }\end{array}$ & ${ }^{*}$ CMSD & $\begin{array}{l}\text { COPD history } \\
\text { (years) }\end{array}$ \\
\hline \multicolumn{9}{|c|}{ Studies with sputum test } \\
\hline Cao [38] & $\begin{array}{l}\text { Xingtai, } \\
\text { Hebei }\end{array}$ & NS & $\begin{array}{l}\text { T: } 40 / 40 \\
\text { C: } 40 / 40\end{array}$ & $\begin{array}{l}\text { T: } 23 / 17 \\
\text { C: } 24 / 16\end{array}$ & $\begin{array}{l}\text { T: } 56 \pm 13 \\
\text { C: } 55 \pm 14\end{array}$ & NS & NS & NS \\
\hline Che et al. [40] & $\begin{array}{l}\text { Shenyang, } \\
\text { Liaoning }\end{array}$ & NS & $\begin{array}{l}\text { T: } 25 / 23 \\
\text { C: } 25 / 23\end{array}$ & $\begin{array}{l}\text { T: } 18 / 7 \\
\mathrm{C}: 16 / 9\end{array}$ & $\begin{array}{l}\mathrm{T}: 65.6 \pm \mathrm{NS} \\
\mathrm{C}: 64.5 \pm \mathrm{NS}\end{array}$ & $\begin{array}{c}\text { T: IIA: } 11, \text { IIB: } 10 \\
\text { III: } 4 \\
\text { C: IIA: } 10, \text { IIB: } 12, \\
\text { III: } 3\end{array}$ & NS & $\begin{array}{l}\mathrm{T}: 14 \pm 8 \\
\mathrm{C}: 15 \pm 7.6\end{array}$ \\
\hline Du et al. [16] & $\begin{array}{l}\text { Shiyan, } \\
\text { Hubei }\end{array}$ & Out & $\begin{array}{l}\text { T: } 18 / 18 \\
\text { C: } 18 / 18\end{array}$ & $\begin{array}{l}\text { T: } 16 / 2 \\
\text { C: } 15 / 3\end{array}$ & $\begin{array}{l}\mathrm{T}: 58.61 \pm 10.69 \\
\mathrm{C}: 58.44 \pm 9.54\end{array}$ & $\begin{array}{l}\text { T: }\|A: 7,\| B: 6,\|1\|: 2 \\
\text { C: }\|A: 6,\| B: 7,\|1\|: 1\end{array}$ & NS & NS \\
\hline Huang [18] & $\begin{array}{c}\text { Fangcheng } \\
\text { gang, } \\
\text { Guangxi }\end{array}$ & Out & $\begin{array}{l}\mathrm{T}: 30 / 30 \\
\mathrm{C}: 30 / 30\end{array}$ & $\begin{array}{l}\text { T: } 16 / 14 \\
\mathrm{C}: 17 / 13\end{array}$ & $\begin{array}{l}\mathrm{T}: 67.38 \pm 5.66 \\
\mathrm{C}: 65.25 \pm 6.13\end{array}$ & $\begin{array}{c}\text { T: IIA: } 12, \| B: 14 \\
\text { III: } 4 \\
\text { C: IIA:10, IIB: } 14, \\
\text { III: } 6\end{array}$ & NS & NS \\
\hline Lu et al. [20] & $\begin{array}{l}\text { Fuzhou, } \\
\text { Fujian }\end{array}$ & Out & $\begin{array}{l}\mathrm{T}: 30 / 30 \\
\mathrm{C}: 30 / 30\end{array}$ & $\begin{array}{l}\mathrm{T}: 25 / 5 \\
\mathrm{C}: 23 / 7\end{array}$ & $\begin{array}{l}\mathrm{T}: 64.6 \pm \mathrm{NS} \\
\mathrm{C}: 63.8 \pm \mathrm{NS}\end{array}$ & $\begin{array}{l}\text { T: II } 30 \\
\text { C: II } 30\end{array}$ & $\begin{array}{l}\text { Deficiency of } \\
\text { Spleen Qi }\end{array}$ & $\begin{array}{l}\mathrm{T}: 11.2 \pm \mathrm{NS} \\
\mathrm{C}: 10.6 \pm \mathrm{NS}\end{array}$ \\
\hline Wang et al. [31] & $\begin{array}{l}\text { Hefei, } \\
\text { Anhui }\end{array}$ & NS & $\begin{array}{l}\text { T: } 20 / 20 \\
\text { C: } 20 / 20\end{array}$ & $\begin{array}{l}\text { T: } 18 / 2 \\
\text { C: } 19 / 1\end{array}$ & $\begin{array}{l}\mathrm{T}: 59.4 \pm 7.5 \\
\mathrm{C}: 60.9 \pm 7.9\end{array}$ & $\begin{array}{l}\text { T: IA: } 8, \| B: 9, \text { III: } 3 \\
\text { C: }\|A: 9,\| B: 9, \text { III: } 2\end{array}$ & $\begin{array}{l}\text { Deficiency of Lung } \\
\text { and Spleen Qi }\end{array}$ & $\begin{array}{l}\mathrm{T}: 11.2 \pm 4.1 \\
\mathrm{C}: 11.8 \pm 4.5\end{array}$ \\
\hline Xiao et al. [39] & $\begin{array}{l}\text { Zhanjiang, } \\
\text { Guangdong }\end{array}$ & NS & $\begin{array}{l}\text { T: } 60 / 60 \\
\mathrm{C}: 56 / 56\end{array}$ & $\begin{array}{l}\mathrm{T}: 38 / 22 \\
\mathrm{C}: 35 / 21\end{array}$ & $\begin{array}{l}\mathrm{T}: 66.4 \pm 8.8 \\
\mathrm{C}: 61.2 \pm 6.3\end{array}$ & $\begin{array}{c}\text { T: IIA: } 23 \\
\text { IIB: } 25, \text { III: } 12 \\
\text { C: IIA: } 21 \\
\text { IIB: } 22, \text { III: } 13\end{array}$ & NS & $\begin{array}{l}\mathrm{T}: 17.3 \pm 10.2 \\
\mathrm{C}: 18.5 \pm 9.3\end{array}$ \\
\hline Zhou et al. [25] & $\begin{array}{l}\text { Taiwu, } \\
\text { Shandong }\end{array}$ & Both & $\begin{array}{l}\text { T: } 30 / 30 \\
\text { C: } 30 / 30\end{array}$ & $\begin{array}{l}\text { T: } 22 / 8 \\
\text { C: } 21 / 9\end{array}$ & $\begin{array}{l}\text { T:NS } \\
\text { C:NS }\end{array}$ & $\begin{array}{l}\text { T: IIA: } 30 \\
\text { C: IIA: } 30\end{array}$ & NS & NS \\
\hline \multicolumn{9}{|c|}{ Studies with serum test } \\
\hline Chen et al. [37] & $\begin{array}{l}\text { Wuhan, } \\
\text { Hubei }\end{array}$ & NS & $\begin{array}{l}\text { T: } 36 / 36 \\
\mathrm{C}: 36 / 36\end{array}$ & $\begin{array}{l}\mathrm{T}: 24 / 12 \\
\mathrm{C}: 26 / 10\end{array}$ & $\begin{array}{l}\mathrm{T}: 62.9 \pm 7.3 \\
\mathrm{C}: 62.2 \pm 7.8\end{array}$ & $\begin{array}{l}\text { T:III:25, IV:11 } \\
\text { C:III:26, IV:10 }\end{array}$ & $\begin{array}{l}\text { Deficiency of Lung } \\
\text { and Kidney Qi and } \\
\text { blood stasis }\end{array}$ & $\begin{array}{l}\mathrm{T}: 5.9 \pm 1.7 \\
\mathrm{C}: 6.2 \pm 1.8\end{array}$ \\
\hline Cheng et al. [41] & Chongqing & Both & $\begin{array}{l}\text { T: } 40 / 40 \\
\text { C: } 40 / 40\end{array}$ & $\begin{array}{l}\text { T: } 35 / 5 \\
\text { C: } 33 / 7\end{array}$ & $\begin{array}{l}\mathrm{T}: 83.2 \pm 7.8 \\
\mathrm{C}: 82.7 \pm 5.6\end{array}$ & NS & NS & NS \\
\hline Feng et al. [30] & Beijing & NS & $\begin{array}{l}\text { T: } 46 / 46 \\
\mathrm{C}: 30 / 30\end{array}$ & $\begin{array}{l}\mathrm{T}: 25 / 21 \\
\mathrm{C}: 14 / 16\end{array}$ & $\begin{array}{l}\mathrm{T}: 63.37 \pm 8.99 \\
\mathrm{C}: 62.26 \pm 8.72\end{array}$ & $\begin{array}{l}T:\|A: 24,\| B: 22 \\
C:\|A: 16,\| B: 14\end{array}$ & $\begin{array}{l}\text { Deficiency of Qi } \\
\text { and sputum blood } \\
\text { stasis }\end{array}$ & NS \\
\hline Fu et al. [42] & Tianjin & NS & $\begin{array}{l}\text { T: } 39 / 39 \\
\text { C: } 31 / 31\end{array}$ & $\begin{array}{l}\mathrm{T}: 31 / 31 \\
\mathrm{C}: 31 / 31\end{array}$ & $\begin{array}{c}\mathrm{T}: 60.56 \pm 8.60 \\
\mathrm{C}: 60.21 \pm 10.01\end{array}$ & NS & NS & NS \\
\hline Guo et al. [17] & Tianjin & Out & $\begin{array}{l}\mathrm{T}: 70 / 69 \\
\mathrm{C}: 70 / 61\end{array}$ & $\begin{array}{l}\mathrm{T}: 41 / 28 \\
\mathrm{C}: 39 / 22\end{array}$ & $\begin{array}{c}\mathrm{T}: 60.81 \pm 8.18 \\
\mathrm{C}: 60.51 \pm 11.03\end{array}$ & NS & $\begin{array}{c}\text { Deficiency of Lung } \\
\text { Spleen Kidney Qi } \\
\text { and sputum blood } \\
\text { stasis }\end{array}$ & $\begin{array}{l}\mathrm{T}: 14.56 \pm 6.32 \\
\mathrm{C}: 14.52 \pm 5.96\end{array}$ \\
\hline $\mathrm{Hu}[22]$ & $\begin{array}{l}\text { Lanzhou, } \\
\text { Gansu }\end{array}$ & In & $\begin{array}{l}\text { T: } 35 / 35 \\
\text { C: } 32 / 32\end{array}$ & $\begin{array}{l}\mathrm{T}: 20 / 15 \\
\mathrm{C}: 14 / 18\end{array}$ & $\begin{array}{l}\mathrm{T}: 64.7 \pm 7.5 \\
\mathrm{C}: 63.2 \pm 5.4\end{array}$ & NS & $\begin{array}{l}\text { Yin deficiency of } \\
\text { Lung and Kidney }\end{array}$ & NS \\
\hline Jiang et al. [44] & $\begin{array}{l}\text { Hezhou, } \\
\text { Guangxi }\end{array}$ & NS & $\begin{array}{l}\text { T: } 30 / 30 \\
\text { C: } 30 / 30\end{array}$ & $\begin{array}{l}\mathrm{T}: 20 / 10 \\
\mathrm{C}: 20 / 10\end{array}$ & $\begin{array}{l}\mathrm{T}: 52.3 \pm 10.9 \\
\mathrm{C}: 51.8 \pm 11.1\end{array}$ & NS & NS & $\begin{array}{l}\mathrm{T}: 12.36 \pm 6.2 \\
\mathrm{C}: 12.74 \pm 6.5\end{array}$ \\
\hline Li et al. [36] & $\begin{array}{l}\text { Guangzhou, } \\
\text { Guangdong }\end{array}$ & Both & $\begin{array}{l}\text { T: } 50 / 49 \\
\text { C: } 50 / 48\end{array}$ & $\begin{array}{l}\mathrm{T}: 27 / 22 \\
\mathrm{C}: 28 / 20\end{array}$ & $\begin{array}{l}\mathrm{T}: 66.3 \pm 10.1 \\
\mathrm{C}: 65.9 \pm 9.8\end{array}$ & NS & $\begin{array}{l}\text { Sputum blood } \\
\text { stasis in Lung }\end{array}$ & $\begin{array}{l}\mathrm{T}: 8.9 \pm 5.0 \\
\mathrm{C}: 9.1 \pm 4.8\end{array}$ \\
\hline Li et al. [19] & $\begin{array}{l}\text { Haerbin, } \\
\text { Heilongjiang }\end{array}$ & Out & $\begin{array}{l}\text { T: } 35 / 35 \\
\text { C: } 35 / 35\end{array}$ & $\begin{array}{l}\mathrm{T}: 23 / 12 \\
\mathrm{C}: 24 / 11\end{array}$ & $\begin{array}{l}\mathrm{T}: 63.7 \pm 5.3 \\
\mathrm{C}: 60.1 \pm 5.3\end{array}$ & $\begin{array}{l}\text { T:I:6, ॥A:19, IIB:10 } \\
C: I: 5,\|A: 20,\| B: 10\end{array}$ & $\begin{array}{l}\text { Deficiency of Lung } \\
\text { and Spleen Qi }\end{array}$ & $\begin{array}{l}\mathrm{T}: 15.4 \pm 3.1 \\
\mathrm{C}: 13.1 \pm 4.1\end{array}$ \\
\hline Ou et al. [32] & $\begin{array}{l}\text { Guiyang, } \\
\text { Guizhou }\end{array}$ & Both & $\begin{array}{l}\text { T: } 36 / 30 \\
\text { C: } 34 / 30\end{array}$ & $\begin{array}{l}\mathrm{T}: 23 / 13 \\
\mathrm{C}: 22 / 12\end{array}$ & NS & NS & $\begin{array}{l}\text { Deficiency of Lung } \\
\text { and Spleen Qi }\end{array}$ & NS \\
\hline Shinozuka et al. [26] & $\begin{array}{l}\text { Chiba, } \\
\text { Japan }\end{array}$ & NS & $\begin{array}{l}\text { T: } 17 / 17 \\
\text { C: } 18 / 18\end{array}$ & NS & NS & NS & NS & NS \\
\hline Su et al. [23] & Beijing & NS & $\begin{array}{l}\text { T: } 35 / 35 \\
\text { C: } 37 / 37\end{array}$ & $\begin{array}{l}\mathrm{T}: 16 / 19 \\
\mathrm{C}: 18 / 19\end{array}$ & $\begin{array}{l}\mathrm{T}: 60.6 \pm 8.9 \\
\mathrm{C}: 56.6 \pm 8.8\end{array}$ & NS & $\begin{array}{l}\text { Deficiency of Qi } \\
\text { and sputum blood } \\
\text { stasis }\end{array}$ & NS \\
\hline Tatsumi et al. [24] & $\begin{array}{l}\text { Chiba, } \\
\text { Japan }\end{array}$ & NS & $\begin{array}{l}\text { T: } 34 / 34 \\
\text { C: } 37 / 37\end{array}$ & NS & NS & NS & NS & NS \\
\hline Wang et al. [29] & Shanghai & NS & $\begin{array}{l}\text { T1:109/82 } \\
\text { T2:109/89 } \\
\text { C:113/91 }\end{array}$ & $\begin{array}{l}\text { T1:64/18 } \\
\text { T2:62/27 } \\
\text { C:69/22 }\end{array}$ & $\begin{array}{l}\mathrm{T} 1: 62.43 \pm 9.04 \\
\mathrm{~T} 2: 61.51 \pm 8.79 \\
\mathrm{C}: 62.68 \pm 8.10\end{array}$ & $\begin{array}{c}\text { T1:I:1, II:34, III:45, } \\
\text { IV:2 } \\
\text { T2:I:0, II:42, III:44, } \\
\text { IV:3 } \\
\text { C:I:2, II:34, III:50, } \\
\text { IV:5 }\end{array}$ & $\begin{array}{l}\text { 1.Deficiency of } \\
\text { Qi 2.Deficiency } \\
\text { of Kidney Qi } \\
\text { 3.Deficiency of } \\
\text { Kidney Yang }\end{array}$ & $\begin{array}{l}\mathrm{T} 1: 12.57 \pm 8.39 \\
\mathrm{~T} 2: 12.75 \pm 8.95 \\
\mathrm{C}: 12.43 \pm 9.46\end{array}$ \\
\hline Wang et al. [28] & $\begin{array}{l}\text { Liuyang, } \\
\text { Hunan }\end{array}$ & Both & $\begin{array}{l}\mathrm{T}: 30 / 30 \\
\mathrm{C}: 30 / 30\end{array}$ & $\begin{array}{l}\mathrm{T}: 16 / 14 \\
\mathrm{C}: 18 / 12\end{array}$ & $\begin{array}{l}T: 65.22 \pm 2.45 \\
C: 62.75 \pm 3.66\end{array}$ & $\begin{array}{l}\text { T:I:5,T:II:19, T:III:6 } \\
\text { C:I:5,C:II:20, C:III:5 }\end{array}$ & $\begin{array}{l}\text { Deficiency of Lung } \\
\text { and Kidney }\end{array}$ & NS \\
\hline Xiao et al. [27] & $\begin{array}{l}\text { Guangzhou, } \\
\text { Guangdong }\end{array}$ & NS & $\begin{array}{l}\text { T: } 34 / 34 \\
\text { C: } 31 / 31\end{array}$ & $\begin{array}{l}\mathrm{T}: 26 / 8 \\
\mathrm{C}: 25 / 6\end{array}$ & $\begin{array}{l}\mathrm{T}: 62.3 \pm 7.11 \\
\mathrm{C}: 64.6 \pm 8.62\end{array}$ & $\begin{array}{l}\text { T:I:6, II:25, III:3 } \\
\text { C:I:7, II:21, III:3 }\end{array}$ & $\begin{array}{l}\text { Yin deficiency of } \\
\text { Lung and Kidney }\end{array}$ & NS \\
\hline Xiong et al. [34] & Shenzhen & Both & $\begin{array}{l}\text { T: } 30 / 30 \\
\text { C: } 30 / 30\end{array}$ & $\begin{array}{l}\mathrm{T}: 20 / 10 \\
\mathrm{C}: 21 / 9\end{array}$ & $\begin{array}{l}\mathrm{T}: 72 \pm \mathrm{NS} \\
\mathrm{C}: 71 \pm \mathrm{NS}\end{array}$ & NS & $\begin{array}{l}\text { Deficiency of Lung } \\
\text { and Kidney }\end{array}$ & NS \\
\hline
\end{tabular}


Citation: Miao Q, Cong X, Du Y, Wang B, Qiao CY, et al. (2016) Anti-Inflammatory Effects of Chinese Herbal Medicine on COPD: A Systematic Review. Lung Dis Treat 2: 107. doi:10.4172/2472-1018.1000107

Page 4 of 11

\begin{tabular}{|c|c|c|c|c|c|c|c|c|}
\hline Zhang et al. [33] & $\begin{array}{c}\text { Zhengzhou, } \\
\text { Henan }\end{array}$ & NS & $\begin{array}{l}\text { T: } 35 / 34 \\
\text { C: } 35 / 34\end{array}$ & $\begin{array}{l}\text { T:NS } \\
\mathrm{C}: 27 / 8\end{array}$ & $\begin{array}{c}\text { T:NS } \\
\text { C: } 61.77 \pm 10.18\end{array}$ & NS & $\begin{array}{c}\text { 1.Deficiency of } \\
\text { Lung and Spleen } \\
\text { Qi 2.Deficiency of } \\
\text { Lung and Kidney } \\
\text { Qi 3.Deficiency of } \\
\text { Qi and Yin }\end{array}$ & NS \\
\hline Zhao et al. [35] & $\begin{array}{l}\text { Xinxiang, } \\
\text { Henan }\end{array}$ & NS & $\begin{array}{l}\text { T: } 30 / 30 \\
\text { C: } 30 / 30\end{array}$ & $\begin{array}{l}\mathrm{T}: 16 / 14 \\
\mathrm{C}: 18 / 12\end{array}$ & $\begin{array}{l}\mathrm{T}: 72.68 \pm 7.18 \\
\mathrm{C}: 72.18 \pm 7.36\end{array}$ & NS & $\begin{array}{l}\text { Deficiency of Lung } \\
\text { and Kidney Qi }\end{array}$ & NS \\
\hline Zheng [43] & $\begin{array}{l}\text { Tangshan, } \\
\text { Hebei }\end{array}$ & Both & $\begin{array}{l}\text { T: } 45 / 45 \\
\text { C: } 45 / 45\end{array}$ & $\begin{array}{l}\mathrm{T}: 30 / 15 \\
\mathrm{C}: 28 / 17\end{array}$ & $\begin{array}{l}\mathrm{T}: 54.3 \pm 4.7 \\
\mathrm{C}: 55.2 \pm 4.1\end{array}$ & NS & NS & $\begin{array}{l}\mathrm{T}: 11.3 \pm 4.3 \\
\mathrm{C}: 10.9 \pm 4.8\end{array}$ \\
\hline Zhong et al. [21] & Shenzhen & Out & $\begin{array}{l}\text { T: } 35 / 35 \\
\text { C: } 35 / 35\end{array}$ & $\begin{array}{l}\mathrm{T}: 20 / 15 \\
\mathrm{C}: 21 / 14\end{array}$ & $\begin{array}{l}\mathrm{T}: 68.3 \pm 9.8 \\
\mathrm{C}: 68.2 \pm 9.5\end{array}$ & NS & $\begin{array}{l}\text { Deficiency of Lung } \\
\text { and Kidney }\end{array}$ & NS \\
\hline
\end{tabular}

Table 1: The characteristics of each study.

3886 Records identified through database searches:

Pubmed, EMBASE, Scopus,

CINAHI, Cochrane \& CNKI, CQVIP, WANFANG

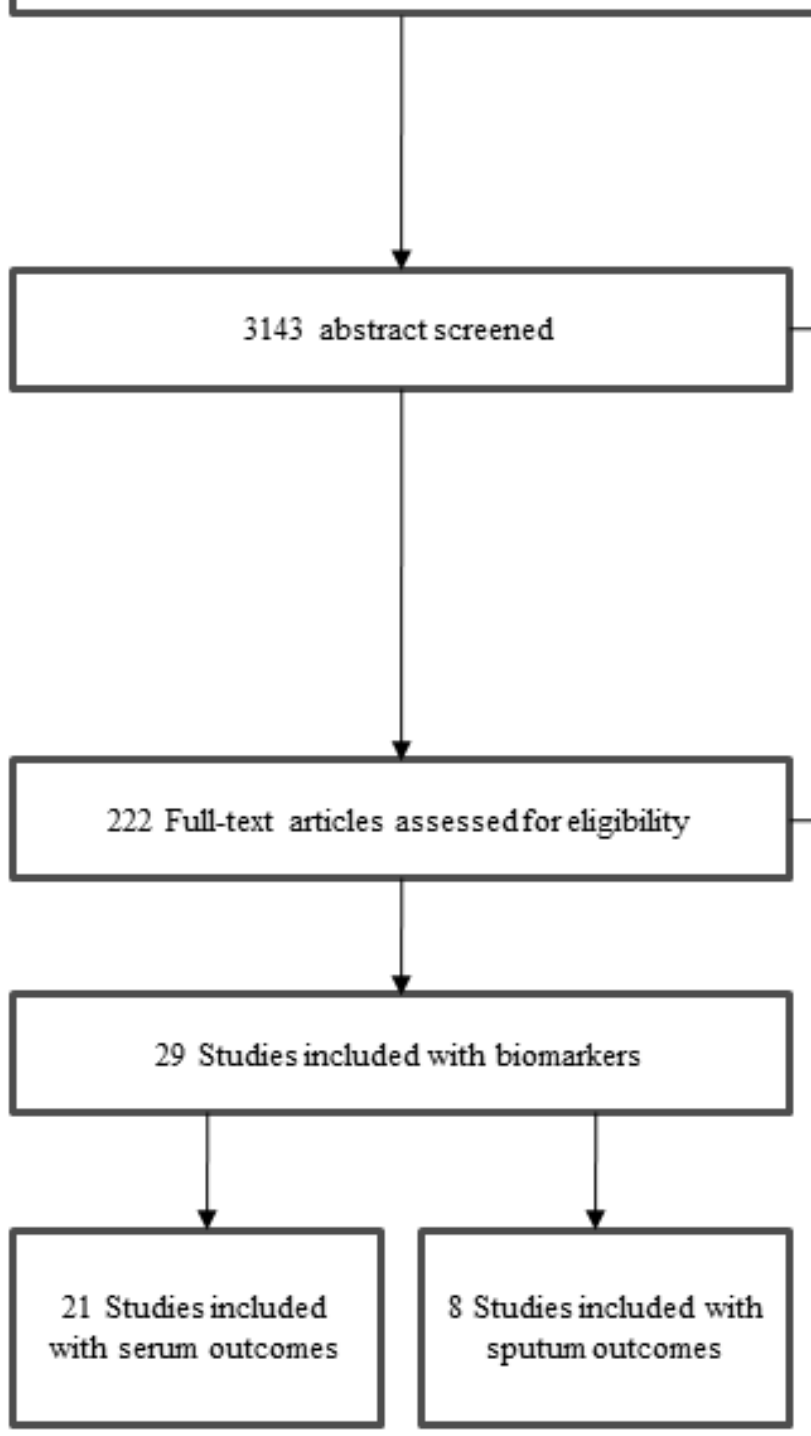

743 duplicates removed

\section{Records excluded}

1. Not RCTs $(\mathrm{n}=270)$

2. Patients not COPD or complicated respiratory failure, heart failure, pulmonary hypertension and cor pulmonale $(\mathrm{n}=333)$

3. Case report $(\mathrm{n}=17)$

4. Survey and retrospective study $(\mathrm{n}=36)$

5. Western drugs or nursing care or health education ( $\mathrm{n}=94$ )

6. Outcomes not biomarkers $(\mathrm{n}=965)$

7. Animal experiments $(\mathrm{n}=212)$

8. Overview $(\mathrm{n}=807)$

9. Other $\mathrm{CM}$ therapy $(\mathrm{n}=187)$

193 Full-text articles excluded, with reason:

1. CHM was administrated by IV drop, enema and inhalation $(\mathrm{n}=81)$

2. Not cytokine outcomes $(\mathrm{n}=66)$

3. Oral CHM for ECOPD or Not clarify the course of COPD ( $n=43$ )

4. Others: $\mathrm{CHM}$ Vs $\mathrm{CHM}(\mathrm{n}=1)$ and not for $\mathrm{CHM}$ $(\mathrm{n}=2)$

Figure 1: The process of selection of studies. 
Citation: Miao Q, Cong X, Du Y, Wang B, Qiao CY, et al. (2016) Anti-Inflammatory Effects of Chinese Herbal Medicine on COPD: A Systematic Review. Lung Dis Treat 2: 107. doi:10.4172/2472-1018.1000107

Page 5 of 11

\begin{tabular}{|c|c|c|c|c|c|c|}
\hline \multirow{2}{*}{$\begin{array}{l}\text { First author, } \\
\text { date }\end{array}$} & \multicolumn{4}{|l|}{ Intervention } & \multirow[b]{2}{*}{ *TD } & \multirow[b]{2}{*}{ outcomes } \\
\hline & $\begin{array}{l}\text { Formula name (form) } \\
\text { /Ingredients/quantitative }\end{array}$ & $\begin{array}{l}\text { Dosage } \\
\text { regimen }\end{array}$ & $\begin{array}{l}\text { Qualitative } \\
\text { testing }\end{array}$ & $\begin{array}{l}\text { Plus } \\
\text { bronchodilators }\end{array}$ & & \\
\hline \multicolumn{7}{|c|}{ Studies with sputum test } \\
\hline Cao [38] & $\begin{array}{l}\text { Xiaoqinglong Decoction } \\
\text { Baishao10 g, Banxia10 g, Gancao10 g, Ganjiang10 g, Guizhi10 g, } \\
\text { Mahuang10 g, Wuweizi10 g, Xixin10 g }\end{array}$ & $\begin{array}{l}1 \text { Dose, } \\
\text { BID }\end{array}$ & Hospital & $\begin{array}{l}\text { Salmeterol/ } \\
\text { fluticasone }\end{array}$ & 2 mths & $\begin{array}{l}\text { Lung function, } \\
\text { *MMP-9 }\end{array}$ \\
\hline Che et al. [40] & $\begin{array}{l}\text { ZhikeQingfei Oral liquid ( } 10 \mathrm{ml} \text { oral liquid contains) } \\
\text { Banlangen } 1.67 \mathrm{~g} \text {, Gancao } 1.67 \mathrm{~g} \text {, Huangqi1 } 67 \mathrm{~g} \text {, Huangqin } 1.67 \mathrm{~g} \text {, } \\
\text { Jinyinhua1.67 g, Jiegeng } 0.83 \mathrm{~g} \text {, Kuandonghua0.67 g, Lianqiao0.83 } \\
\text { g, Mahuang0.5 g, } \\
\text { Pipaye } 0.83 \mathrm{~g} \text {, Yuxingcao } 0.83 \mathrm{~g} \text {, Ziwan } 0.67 \mathrm{~g}\end{array}$ & $\begin{array}{l}20 \mathrm{ml} \\
\text { TID }\end{array}$ & Hospital & Bronchodilators & 6 wks & Lung function \\
\hline Du et al. [16] & $\begin{array}{l}\text { Yinxingye extract tablet: } \\
\text { Ginkgo biloba extract }\end{array}$ & $\begin{array}{l}2 \text { Tablets, } \\
\text { TID }\end{array}$ & Hospital & No & $2 \mathrm{mths}$ & Lung function \\
\hline Huang [18] & $\begin{array}{l}\text { BufeiYiyangHuatangranules: } \\
\text { Bajitian, Chuanbeimu, Dangshen, Duzhong, Gouqizi, } \\
\text { Huanghuadaoshuilian, Huangqi, Jupi, Maimendong, Pipaye, } \\
\text { Sangbaipi, Wuweizi, Yipichou, Yuxingcao ,Ziwan }\end{array}$ & $20 \mathrm{~g}, \mathrm{BID}$ & Hospital & Bronchodilators & 6 mths & NS \\
\hline Lu et al. [20] & $\begin{array}{l}\text { Liu Junzi Decoction contains ( } 150 \mathrm{ml} \text { ): } \\
\text { Baizhu } 9 \mathrm{~g} \text {, Banxia } 9 \mathrm{~g} \text {, Chenpi } 6 \mathrm{~g} \text {, Dangshen } 20 \mathrm{~g} \text {, Fuling } 15 \mathrm{~g}\end{array}$ & $\begin{array}{l}150 \mathrm{ml} \\
\mathrm{BID}\end{array}$ & Hospital & Bronchodilators & 3 mths & $\begin{array}{l}\text { Sputum *HDACs } \\
\text { activity }\end{array}$ \\
\hline $\begin{array}{l}\text { Wang et al. } \\
\qquad[31]\end{array}$ & $\begin{array}{l}\text { YifeiJianpi Fang: Decoction (1 dose contains): } \\
\text { Baizhu } 15 \mathrm{~g} \text {, Banxia } 15 \mathrm{~g} \text {, Chenpi } 10 \mathrm{~g} \text {,Dangshen } 15 \mathrm{~g} \text {, Dilong } \\
8 \mathrm{~g} \text {, Fangfeng } 10 \mathrm{~g} \text {, Fuling } 15 \mathrm{~g} \text {, Gancao } 10 \mathrm{~g} \text {, Huangqi } 30 \mathrm{~g} \text {, } \\
\text { Kuandonghua } 10 \mathrm{~g}\end{array}$ & $\begin{array}{l}1 \text { Dose, } \\
\text { BID }\end{array}$ & Hospital & Bronchodilators & 2 mths & Lung function \\
\hline Xiao et al. [39] & $\begin{array}{l}\text { ManzhiKechuanling: Oral liquid: } \\
\text { Baizhu, Banxia, Buguzhi, Chuanbeimu, Ejiao, Fuling, Gancao, Gejie, } \\
\text { Hetaorou, Huangqi, Lujiaojia, Renshen, Shanyao, Taoren, Yimucao, } \\
\text { Ziheche, Zisuzi }\end{array}$ & $\begin{array}{l}10 \mathrm{ml} \\
\text { BID }\end{array}$ & Hospital & Bronchodilators & 2 mths & MMP-9 \\
\hline $\begin{array}{l}\text { Zhou et al. } \\
\quad[25]\end{array}$ & $\begin{array}{l}\text { Feisaitong Heji: Oral liquid ( } 100 \mathrm{ml} \text { contains): } \\
\text { Chantui } 9 \mathrm{~g} \text {, Danshen } 30 \mathrm{~g} \text {, Dilong } 12 \mathrm{~g} \text {, Gancao } 6 \mathrm{~g} \text {, Huangqi } 30 \mathrm{~g} \text {, } \\
\text { Jiegeng } 9 \mathrm{~g} \text {, Jinyinhua } 18 \mathrm{~g} \text {, Laifuzi } 12 \mathrm{~g} \text {, Shashen } 18 \mathrm{~g} \text {, Yiyiren } 30 \\
\text { g, Zisuye } 9 \mathrm{~g}\end{array}$ & $\begin{array}{l}100 \mathrm{ml} \\
\text { TID }\end{array}$ & Hospital & Bronchodilators & $1 \mathrm{mth}$ & $\begin{array}{l}\text { Lung function, } \\
\text { Syndromes }\end{array}$ \\
\hline \multicolumn{7}{|c|}{ Studies with serum test } \\
\hline $\begin{array}{l}\text { Chen et al. } \\
\text { [37] }\end{array}$ & $\begin{array}{l}\text { BufeiHuoxue Decoction (dose contains): } \\
\text { Banxia } 10 \mathrm{~g} \text {, Chuanxiong } 15 \mathrm{~g} \text {, Danggui } 10 \mathrm{~g} \text {, Dihuang } 20 \mathrm{~g} \text {, Dilong } \\
10 \mathrm{~g} \text {, Fuling } 15 \mathrm{~g} \text {, Gancao } 6 \mathrm{~g} \text {, Honghua } 6 \mathrm{~g} \text {, Huajuhong } 15 \mathrm{~g} \text {, } \\
\text { Huangjing } 20 \mathrm{~g} \text {, Huangqi } 30 \mathrm{~g} \text {, Laifuzi } 15 \mathrm{~g} \text {, Taoren } 10 \mathrm{~g} \text {, Wuweiz } 6 \\
\text { g, Xiyangshen } 10 \mathrm{~g} \text {, Yinyanghuo } 10 \mathrm{~g}\end{array}$ & $\begin{array}{l}1 \text { Dose, } \\
\text { QD }\end{array}$ & Hospital & No & 3 mths & $\begin{array}{l}\text { Lung function } \\
{ }^{*} 6 \mathrm{MWD},{ }^{*} \mathrm{CAT}\end{array}$ \\
\hline $\begin{array}{l}\text { Cheng et al. } \\
\qquad[41]\end{array}$ & $\begin{array}{l}\text { SuhuangZhike Capsule: } \\
\text { Dilong, Mahuang, Niubangzi, Qianhu, Wuweizi, Zisuye }\end{array}$ & $\begin{array}{l}3 \text { Capsules, } \\
\text { TID }\end{array}$ & $\begin{array}{l}\text { Yangtze River } \\
\text { Pharmaceutical } \\
\text { Group }\end{array}$ & No & $1 \mathrm{mth}$ & Lung function \\
\hline $\begin{array}{l}\text { Feng et al. } \\
\text { [30] }\end{array}$ & $\begin{array}{l}\text { Qi-replenishing Blood-activating and Phlegm-removing Decoction: } \\
\text { Huangqi, Shuizhi, Banxia, Dilong }\end{array}$ & $\begin{array}{l}1 \text { Dose, } \\
\text { QD }\end{array}$ & Hospital & Bronchodilators & 3 mths & Quality of life, \\
\hline Fu et al. [42] & $\begin{array}{l}\text { Bufei granules: } \\
\text { Chenpi, Dangshen, Dihuang, Mahuang, Shanzhuyu, Ziwan }\end{array}$ & $\begin{array}{l}16 \mathrm{~g} \\
\mathrm{BID}\end{array}$ & Hospital & No & $1 \mathrm{mth}$ & *TCM syndromes \\
\hline Guo et al. [17] & $\begin{array}{l}\text { Bufei granules: } \\
\text { Banxia, Chenpi, Chishao, Danggui, Dangshen, Dihuang, Gancao, } \\
\text { Huangqin, Mahuang, Shanzhuyu, Ziwan }\end{array}$ & $\begin{array}{l}16 \mathrm{~g} \\
\mathrm{BID}\end{array}$ & Hospital & No & 3 mths & NS \\
\hline $\mathrm{Hu}[22]$ & $\begin{array}{l}\text { Jiajianbufei Decoction: } \\
\text { Baibu10 g, Bajitian15 g, Buguzhi15 g, Chenpi } 12 \mathrm{~g} \text {, } \\
\text { Dangshen } 15 \mathrm{~g} \text {, Danshen, Huangqi } 20 \mathrm{~g} \text {, Jiegeng } 10 \mathrm{~g} \text {, Maidong10 } \\
\text { g, Sangbaipi10 g, Tusizi10 g, Xuanshen } 10 \mathrm{~g}\end{array}$ & $\begin{array}{l}1 \text { Dose, } \\
\text { QD }\end{array}$ & Hospital & $\begin{array}{l}\text { Inhaled albuterol } \\
\& \text { Aminophylline } \\
\text { Sustained } \\
\text { release tablets }\end{array}$ & 2 mths & Lung function \\
\hline $\begin{array}{l}\text { Jiang et al. } \\
\quad[44]\end{array}$ & $\begin{array}{l}\text { BufeiNashen Decoction: } \\
\text { Every dose contains: Chenxiang10 g, Dangshen } 20 \mathrm{~g} \text {, Dihuang } 20 \mathrm{~g} \text {, } \\
\text { Fuling15 g, Gancao } 6 \mathrm{~g} \text {, Gejie1 pair, Huangqi } 20 \mathrm{~g} \text {, Sangbaipi } 15 \mathrm{~g} \text {, } \\
\text { Wuweizi } 15 \mathrm{~g} \text {, Ziwan } 15 \mathrm{~g}\end{array}$ & $\begin{array}{l}2 \text { Doses, } \\
\text { QD }\end{array}$ & Hospital & $\begin{array}{l}\text { Salmeterol/ } \\
\text { fluticasone }\end{array}$ & 2 wks & Lung function \\
\hline Li et al. [36] & $\begin{array}{l}\text { SanziYangqin Decoction \&TaohongSiwu Decoction: } \\
\text { Baijiezi10 g, Baishao15 g, Chuanxiong15 g, Danggui10 g, } \\
\text { Dihuang10 g, Honghua10 g, Laifuzi10 g, Taoren15 g, Zisuzi15 g }\end{array}$ & $\begin{array}{l}150 \mathrm{ml} \\
\text { QD }\end{array}$ & Hospital & $\begin{array}{l}\text { Aminophylline } \\
\text { Sustained } \\
\text { release tablets }\end{array}$ & $1 \mathrm{mth}$ & Lung function \\
\hline Li et al. [19] & $\begin{array}{l}\text { YiqiJianpiHuatanFangDecoction: } \\
\text { Baizhu, Banxia, Chenpi, Dangshen, Huangqi, Xingren }\end{array}$ & $\begin{array}{l}1 \text { Dose, } \\
\text { QD }\end{array}$ & $\begin{array}{l}\text { Jiangyin } \\
\text { Tianjiang } \\
\text { Pharmaceutical } \\
\text { Co }\end{array}$ & Bronchodilators & 3 mths & SGRQ \\
\hline Ou et al. [32] & $\begin{array}{l}\text { JiajianBufei Decoction: } \\
\text { Huangqi } 20 \text { g, Danshen15 g, Dangshen10 g, Buguzhi15 g, Baibu } 10 \\
\text { g, Sangbaipi10 g }\end{array}$ & $\begin{array}{l}300 \mathrm{ml} \\
\text { QD }\end{array}$ & Hospital & Bronchodilators & 3 mths & $\begin{array}{l}\text { Lung function, } \\
\text { TCM syndromes }\end{array}$ \\
\hline $\begin{array}{l}\text { Shinozuka et } \\
\text { al. [26] }\end{array}$ & Hochuekkito extract (BuzhongYiqi Tang): $7.5 \mathrm{~g}$ extract & $\begin{array}{l}2.5 \mathrm{~g} \\
\text { TID }\end{array}$ & NS & Bronchodilators & 6 mths & Lung function \\
\hline Su et al. [23] & $\begin{array}{l}\text { Feikang Granules: } \\
\text { Huangqi, Haigeqiao }\end{array}$ & $\begin{array}{l}10 \mathrm{~g} \\
\text { TID }\end{array}$ & Hospital & No & 3 mths & Lung function \\
\hline
\end{tabular}


Citation: Miao Q, Cong X, Du Y, Wang B, Qiao CY, et al. (2016) Anti-Inflammatory Effects of Chinese Herbal Medicine on COPD: A Systematic Review. Lung Dis Treat 2: 107. doi:10.4172/2472-1018.1000107

Page 6 of 11

\begin{tabular}{|c|c|c|c|c|c|c|}
\hline \multirow{2}{*}{$\begin{array}{l}\text { First author, } \\
\text { date }\end{array}$} & \multicolumn{4}{|l|}{ Intervention } & \multirow[b]{2}{*}{ *TD } & \multirow[b]{2}{*}{ outcomes } \\
\hline & $\begin{array}{l}\text { Formula name (form) } \\
\text { /Ingredients/quantitative }\end{array}$ & $\begin{array}{l}\text { Dosage } \\
\text { regimen }\end{array}$ & $\begin{array}{l}\text { Qualitative } \\
\text { testing }\end{array}$ & $\begin{array}{l}\text { Plus } \\
\text { bronchodilators }\end{array}$ & & \\
\hline $\begin{array}{l}\text { Tatsmi et al. } \\
\text { [24] }\end{array}$ & Hochuekkito extract(BuzhongYiqi Tang): $7.5 \mathrm{~g}$ extract & $\begin{array}{l}2.5 \mathrm{~g} \\
\text { TID }\end{array}$ & NS & Bronchodilators & 6 mths & $\begin{array}{l}\text { Lung function, } \\
\text { *SGRQ }\end{array}$ \\
\hline $\begin{array}{l}\text { Wang et al. } \\
\text { [29] }\end{array}$ & $\begin{array}{l}\text { BushenFangchuan tablet } \\
\text { BushenYiqi granule }\end{array}$ & $\begin{array}{l}5 \text { tablets, } \\
\text { TID \&1 Bag, } \\
\text { BID }\end{array}$ & $\begin{array}{l}\text { The } 2 \text { nd TCM } \\
\text { manufactory } \\
\text { of Taiji Group } \\
\text { \&Tianjiang } \\
\text { Pharmacy } \\
\text { company Ltd }\end{array}$ & Inhaled albuterol & 6 mths & $\begin{array}{l}6 \text { MWD, } \\
\text { lung function, } \\
\text { *AEF, } \\
\text { *BODE SGRQ }\end{array}$ \\
\hline $\begin{array}{l}\text { Wang et al. } \\
\text { [28] }\end{array}$ & $\begin{array}{l}\text { FufangYishenDuqi Capsule }(0.7 \mathrm{~g}) \text { : } \\
\text { Dangshen } 15 \mathrm{~g} \text {, Wuweizi10 g, Maidong } 10 \mathrm{~g} \text {, Baibu10 g } \\
\text {, Xingren10 g, Duzhong10 g, Mahuang } 6 \mathrm{~g} \text {, Yiyiren } 20 \mathrm{~g} \text {, } \\
\text { Kuandonghua10 g, Zisuzi } 10 \mathrm{~g} \text {, Chenxiang } 3 \mathrm{~g} \text {, Baijiezi } 15 \mathrm{~g} \text {, } \\
\text { Baishao10 g, Baihe,10 g Houpo10 g, Shanzhuyu } 15 \mathrm{~g}\end{array}$ & $\begin{array}{l}0.7 \mathrm{~g} \\
\text { TID }\end{array}$ & Hospital & $\begin{array}{l}\text { Tiotropium } \\
\text { Bromide Powder }\end{array}$ & 3 mths & $\begin{array}{l}\text { SGRQ, } \\
\text { TCM syndromes }\end{array}$ \\
\hline Xiao et al. [27] & $\begin{array}{l}\text { ZhenqiFuzheng granules: } \\
\text { Huangqi, Nvzhenzi }\end{array}$ & $\begin{array}{l}15 \mathrm{~g} \\
\mathrm{BID}\end{array}$ & $\begin{array}{l}\text { Xiuzheng } \\
\text { Pharmaceutical } \\
\text { Group }\end{array}$ & Bronchodilators & 3 mths & $\begin{array}{l}\text { Lung function, } \\
\text { TCM syndromes }\end{array}$ \\
\hline $\begin{array}{l}\text { Xiong et al. } \\
\text { [34] }\end{array}$ & $\begin{array}{l}\text { Shenge powder: } \\
\text { Gejie, Renshen }\end{array}$ & $\begin{array}{l}5 \mathrm{~g} \\
\text { BID }\end{array}$ & Hospital & $\begin{array}{l}\text { Salmeterol/ } \\
\text { fluticasone } \\
\text { propionate }\end{array}$ & 6 mths & Lung function \\
\hline $\begin{array}{l}\text { Zhang et al. } \\
\qquad[33]\end{array}$ & Fixed prescription of TCM therapy: Granules: & $\begin{array}{l}3 \text { Bags, } \\
\text { TID }\end{array}$ & $\begin{array}{l}\text { Jiangyin } \\
\text { Tianjiang } \\
\text { Pharmaceutical } \\
\text { Co. }\end{array}$ & No & 6 mths & $\begin{array}{l}\text { T-cell subset } \\
\text { numbers }\end{array}$ \\
\hline $\begin{array}{l}\text { Zhao et al. } \\
\quad[35]\end{array}$ & $\begin{array}{l}\text { Bailing Capsule: } \\
\text { Fermentation of cordycepssinensis powder }\end{array}$ & $\begin{array}{l}1.0 \mathrm{~g} \\
\text { TID }\end{array}$ & $\begin{array}{l}\text { East China } \\
\text { Pharmaceutical } \\
\text { Group Limited } \\
\text { Co }\end{array}$ & Inhaled albuterol & 6 mths & $\begin{array}{l}6 \mathrm{MWD} \\
\text { lung function }\end{array}$ \\
\hline Zheng [43] & $\begin{array}{l}\text { Jinshuibao Capsule: } \\
\text { Fermentation of cordycepssinensis powder }\end{array}$ & $\begin{array}{l}3 \text { capsules } \\
(0.99 \mathrm{~g}) \\
\text { TID }\end{array}$ & $\begin{array}{l}\text { Jiangxi } \\
\text { Jiminkexin Group } \\
\text { Co }\end{array}$ & Bronchodilators & 1 mths & NS \\
\hline $\begin{array}{l}\text { Zhong et al. } \\
\quad[21]\end{array}$ & $\begin{array}{l}\text { JiaweiJinshuiLiujunJian Decoction: } \\
\text { Dangshen } 15 \mathrm{~g} \text {, Huangqi } 20 \mathrm{~g} \text {, Banxia } 10 \mathrm{~g} \text {, Danggui } 10 \mathrm{~g} \text {, Dihuang } \\
20 \mathrm{~g} \text {, Fuling } 10 \mathrm{~g} \text {, Chenpi } 10 \mathrm{~g} \text {, Shengjiang } 5 \mathrm{~g} \text {, Gancao } 5 \mathrm{~g} \text {, } \\
\text { Wuweizi } 5 \mathrm{~g}\end{array}$ & $\begin{array}{l}100 \mathrm{ml} \\
\mathrm{BID}\end{array}$ & Hospital & $\begin{array}{l}\text { Aminophylline } \\
\text { Sustained } \\
\text { release tablets }\end{array}$ & 3 mths & $\begin{array}{l}\text { Syndromes, } \\
\text { Amount of } \\
\text { sputum }\end{array}$ \\
\hline
\end{tabular}

Table 2: Intervention of included studies.

were low risk. The Blinding Method in three studies $[17,29,42]$ were low risk; one study [20] had high risk due to its inadequate description of its Blinding Method. The remaining twenty five studies did not clearly describe its methods. Outcome Assessments were low risk in every study. Incomplete Data was either high risk in three studies without using of intention to treat analysis $[17,33,36]$ or unclear in three studies $[21,24,26]$. Two studies were unclear of their Selective Reporting method [24,26]. Other Bias was assessed as low risk in all studies based on baseline data comparison (Figure 2).

\section{Assessment of the quality of grading of recommendation}

The quality of Grading of Recommendation for the serum concentration of IL- 8 , TNF- $\alpha$, TGF- $\beta 1$, IL- 6 , and the sputum concentration of IL-8 was assessed as moderate level. The sputum concentration of TNF- $\alpha$ was assessed as low level due to its small sample size (Table 3).

\section{Outcomes}

In twenty two studies with 1,755 participants, the serum level of IL8 , TNF- $\alpha$, TGF- $\beta 1$, and IL- 6 were analyzed. Sputum levels of IL- 8 and TNF- $\alpha$ were tested and reported in eight studies with 498 participants. Sputum level of IL-10 and MMP-9 was tested in one study [38]. The original data of IL-8, IL- 6 , TNF- $\alpha$, and TGF- $\beta 1$ were not included in four studies, and therefore not included in the meta-analysis $[23,24,26,30]$.
Due to the usage of both Enzyme-Linked Immunosorbent Assay and Radioimmunoassay, the different units were converted and consolidated based on the Wang study before the meta-analysis [29].

\section{Results of serum level of IL-8, TNF- $\alpha$, IL-6 and TGF- $\beta 1$}

The serum level of IL-8 was analyzed in ten studies with 1,020 participants. Wang 2014 [29] was a three-arm clinical trial that compared the control group with two CHM groups. The data from Wang 2014 was used twice. Figure 3 showed that the serum level of IL-8 was significantly reduced in eleven studies with 1,111 patients $[17,21,28,29,32-37,41]$ (MD-1.27, 95\% CI [-1.86, -0.68]) $(\mathrm{p}<0.0001)$.

In eight studies with 648 participants, the serum level of TNF- $\alpha$ was found to be significantly reduced $[17,21,22,27,32,33,36,43]$ (MD -0.72 , 95\% CI [-1.01, -0.43]) $(\mathrm{p}<0.00001)$ (Figure 4).

In five studies with 602 participants, the serum level of IL-6 was found to be significantly reduced $[17,29,41,42,44]$. Figure 5 shows six studies with 693 patients. The data was used from Wang [29] twice (MD-0.75, 95\% CI [-0.90, -0.60]) ( $\mathrm{p}=0.02)$ (Figure 5).

In four studies with 552 participants, the serum level of TGF- $\beta 1$ was found to be significant reduced $[17,19,29,43]$. Figure 3 shows six studies with 643 patients. The data was used from Wang [29] twice (MD -123.62, 95\% CI [-134.33, -122.91]) ( $\mathrm{p}=0.003)$ (Figure 6). 
Citation: Miao Q, Cong X, Du Y, Wang B, Qiao CY, et al. (2016) Anti-Inflammatory Effects of Chinese Herbal Medicine on COPD: A Systematic Review. Lung Dis Treat 2: 107. doi:10.4172/2472-1018.1000107

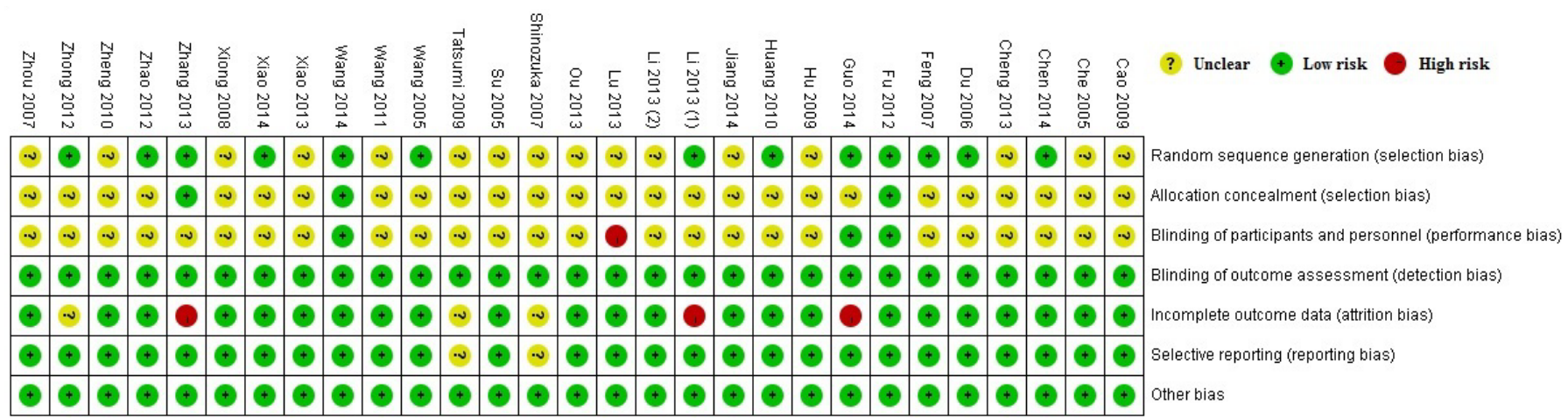

Figure 2: The assessment of risk of bias of 29 studies.

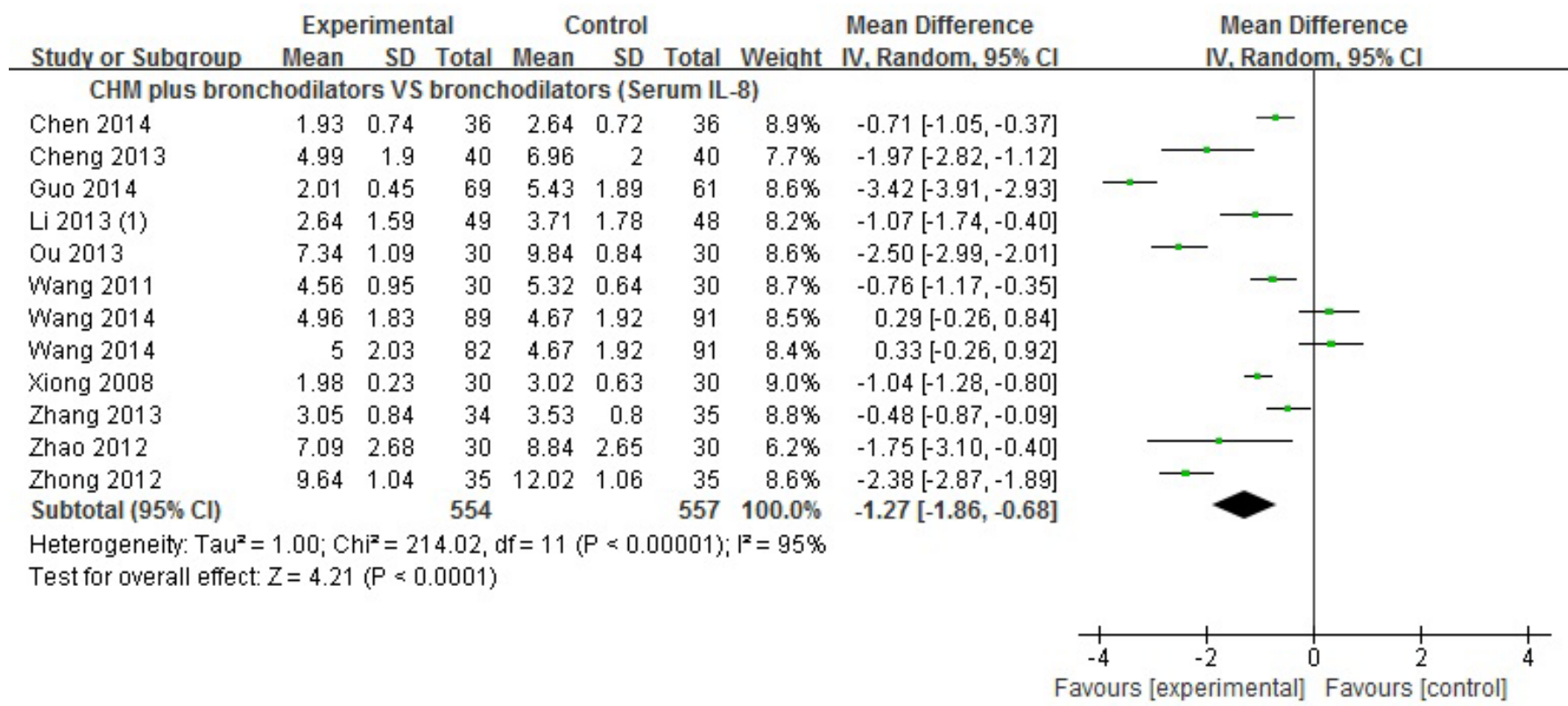

Figure 3: Meta-analysis of CHM plus bronchodilators versus bronchodilators with reduction of serum IL-8 at the end of treatment as the outcome.

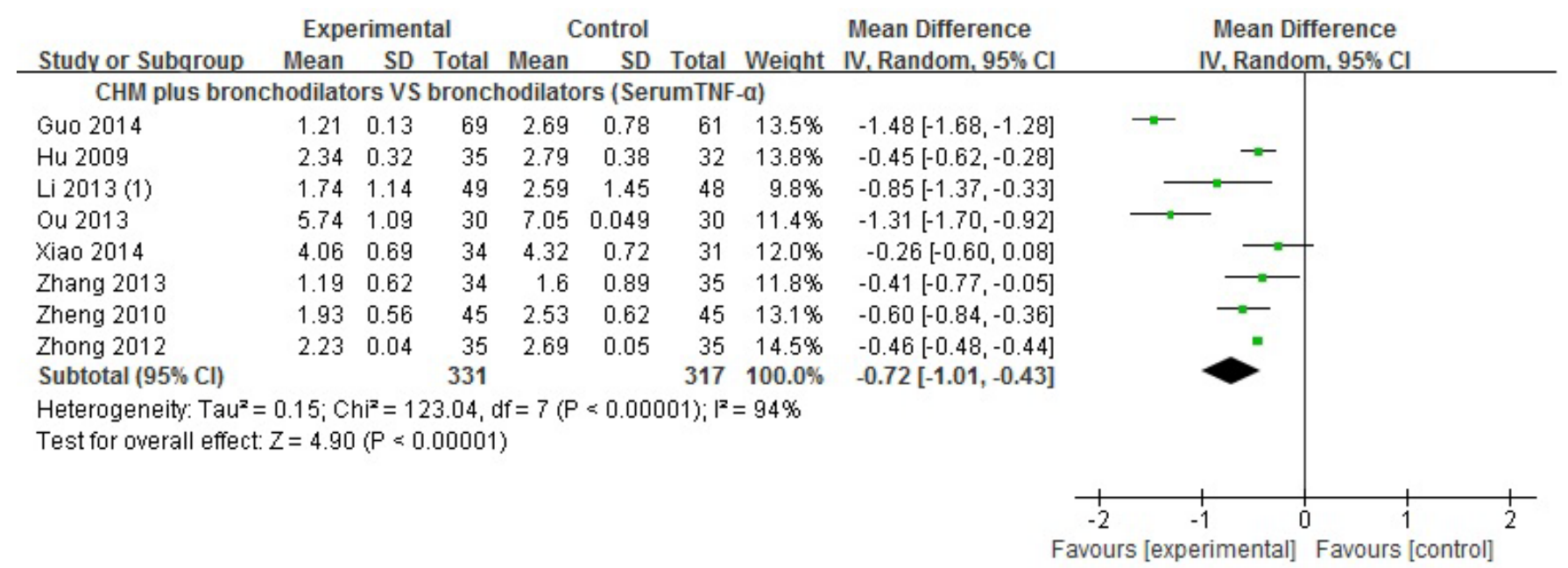

Figure 4: Meta-analysis of CHM plus bronchodilators versus bronchodilators with reduction of serum TNF- $\alpha$ at the end of treatment as the outcome. 
Citation: Miao Q, Cong X, Du Y, Wang B, Qiao CY, et al. (2016) Anti-Inflammatory Effects of Chinese Herbal Medicine on COPD: A Systematic Review. Lung Dis Treat 2: 107. doi:10.4172/2472-1018.1000107

Page 8 of 11

\begin{tabular}{|c|c|c|c|c|c|c|c|c|c|}
\hline \multicolumn{6}{|c|}{ Quality assessment } & \multicolumn{2}{|c|}{ No of patients } & \multirow{2}{*}{$\begin{array}{l}\text { Effect } \\
\text { Absolute }\end{array}$} & \multirow[b]{2}{*}{ Quality } \\
\hline $\begin{array}{l}\text { No of } \\
\text { studies }\end{array}$ & $\begin{array}{l}\text { Risk of } \\
\text { bias }\end{array}$ & Inconsistency & Indirectness & Imprecision & Other considerations & CHM & Control & & \\
\hline \multicolumn{10}{|c|}{ Serum IL-8 (Better indicated by lower values) } \\
\hline 11 & Serious $^{1}$ & Serious $^{2}$ & $\begin{array}{l}\text { No serious } \\
\text { indirectness }\end{array}$ & $\begin{array}{l}\text { No serious } \\
\text { imprecision }\end{array}$ & Reduced effect for $\mathrm{RR}>>1$ or $\mathrm{RR}<<1^{3}$ & 554 & 557 & $\begin{array}{l}\text { MD } 1.27 \text { lower } \\
\text { (1.86 to } 0.68 \text { lower) }\end{array}$ & MODERATE \\
\hline \multicolumn{10}{|c|}{ Serum TNF- $\alpha$ (Better indicated by lower values) } \\
\hline 8 & Serious $^{1}$ & Serious $^{2}$ & $\begin{array}{l}\text { No serious } \\
\text { indirectness }\end{array}$ & $\begin{array}{l}\text { No serious } \\
\text { imprecision }\end{array}$ & Reduced effect for $R R>>1$ or $R R<<1^{3}$ & 331 & 317 & $\begin{array}{l}\text { MD } 0.72 \text { lower } \\
\text { (1.01 to } 0.43 \text { lower) }\end{array}$ & MODERATE \\
\hline \multicolumn{10}{|c|}{ Serum IL-6 (Better indicated by lower values) } \\
\hline 5 & Serious $^{1}$ & Serious $^{2}$ & $\begin{array}{l}\text { No serious } \\
\text { indirectness }\end{array}$ & $\begin{array}{l}\text { No serious } \\
\text { imprecision }\end{array}$ & Reduced effect for $\mathrm{RR}>>1$ or $\mathrm{RR}<<1^{3}$ & 349 & 344 & $\begin{array}{l}\text { MD } 1 \text { lower } \\
\text { (1.87 to } 0.14 \text { lower) }\end{array}$ & MODERATE \\
\hline \multicolumn{10}{|c|}{ Serum TGF- $\beta 1$ (Better indicated by lower values) } \\
\hline 4 & Serious $^{1}$ & Serious $^{2}$ & $\begin{array}{l}\text { No serious } \\
\text { indirectness }\end{array}$ & $\begin{array}{l}\text { No serious } \\
\text { imprecision }\end{array}$ & Reduced effect for $\mathrm{RR}>>1$ or $\mathrm{RR}<<1^{3}$ & 320 & 323 & $\begin{array}{l}\text { MD } 278.66 \text { lower } \\
\text { (460.57 to } 96.75 \text { lower) }\end{array}$ & MODERATE \\
\hline \multicolumn{10}{|c|}{ Sputum IL-8 (Better indicated by lower values) } \\
\hline 7 & Serious $^{1}$ & Serious $^{2}$ & $\begin{array}{l}\text { No serious } \\
\text { indirectness }\end{array}$ & $\begin{array}{l}\text { No serious } \\
\text { imprecision }\end{array}$ & Reduced effect for $\mathrm{RR}>>1$ or $\mathrm{RR}<<1^{3}$ & 221 & 217 & $\begin{array}{l}\text { MD } 0.88 \text { lower } \\
\text { (1.45 to } 0.31 \text { lower) }\end{array}$ & MODERATE \\
\hline \multicolumn{10}{|c|}{ Sputum TNF- $\alpha$ (Better indicated by lower values) } \\
\hline 4 & Serious $^{1}$ & Serious $^{2}$ & $\begin{array}{l}\text { No serious } \\
\text { indirectness }\end{array}$ & Serious $^{4}$ & Reduced effect for $\mathrm{RR}>>1$ or $\mathrm{RR}<<1^{3}$ & 103 & 103 & $\begin{array}{l}\text { SMD } 1.05 \text { lower } \\
\text { (1.97 to } 0.13 \text { lower) }\end{array}$ & LOW \\
\hline \multicolumn{10}{|c|}{$\begin{array}{l}{ }^{1} \text { Allocation concealment in majority of studies was ur } \\
{ }^{2} \text { Heterogeneity was high } \\
\text { 3Severity of COPD and duration of treatment } \\
\text { 4The sample size was small }\end{array}$} \\
\hline
\end{tabular}

Table 3: The quality of grading of recommendation assessment by GRADE pro.

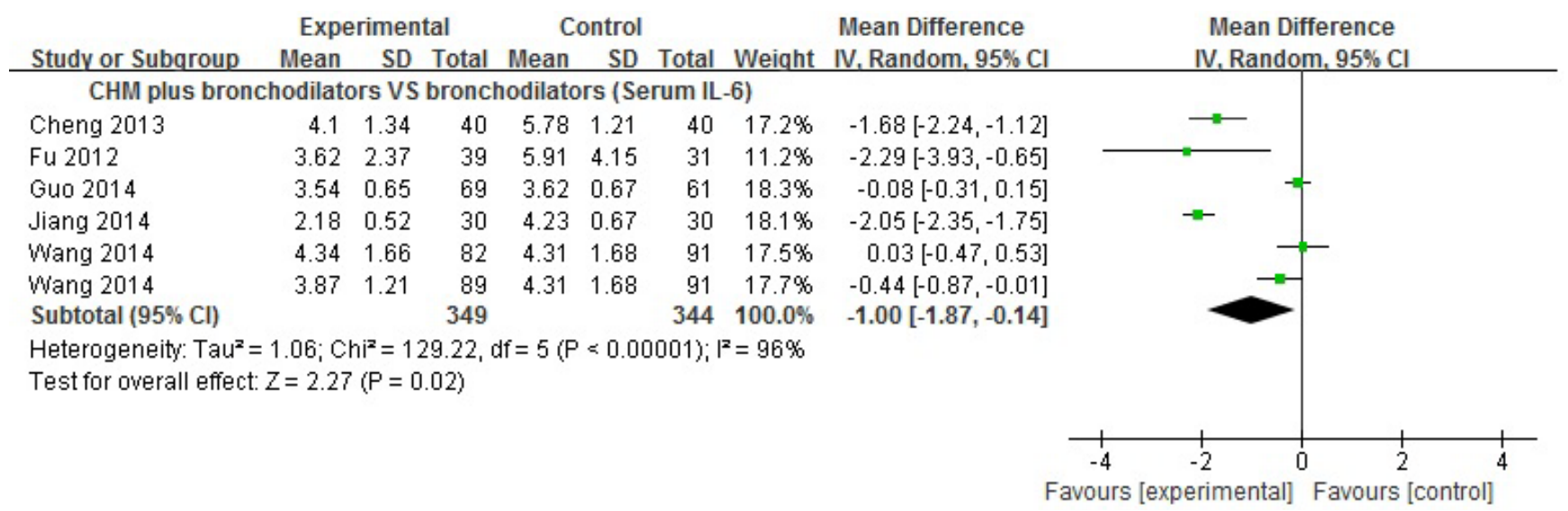

Figure 5: Meta-analysis of CHM plus bronchodilators versus bronchodilators with reduction of serum IL-6 at the end of treatment as the outcome.

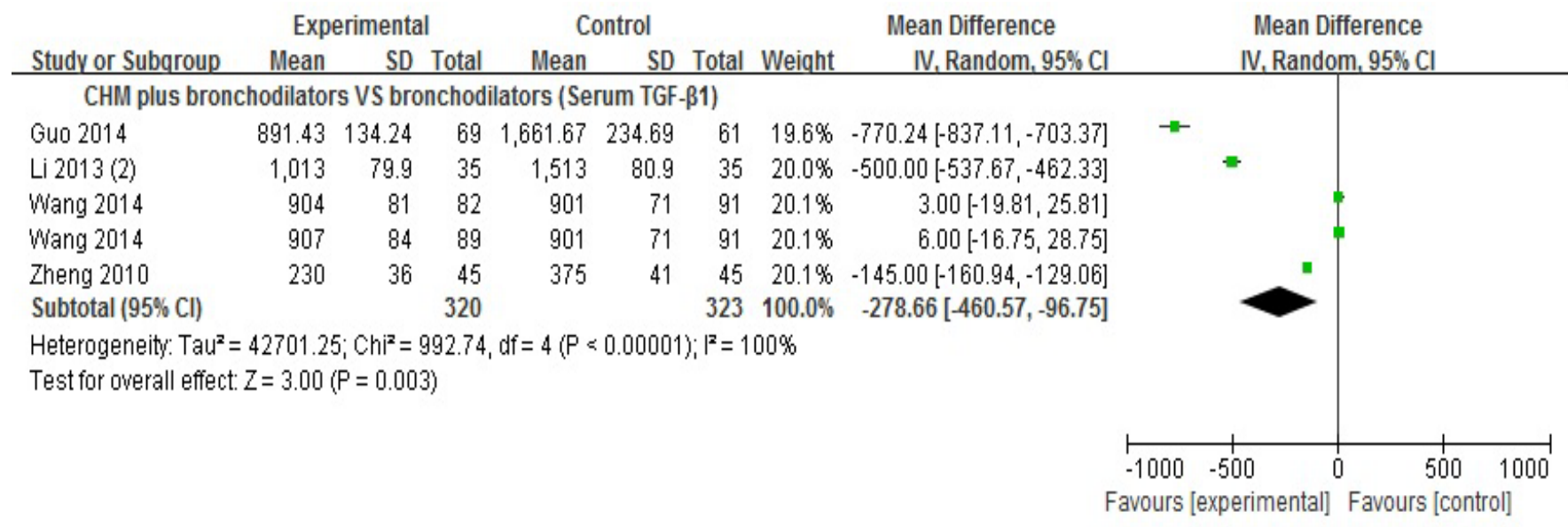

Figure 6: Meta-analysis of CHM plus bronchodilators versus bronchodilators with reduction of serum TGF- $\beta 1$ at the end of treatment as the outcome. 
Citation: Miao Q, Cong X, Du Y, Wang B, Qiao CY, et al. (2016) Anti-Inflammatory Effects of Chinese Herbal Medicine on COPD: A Systematic Review. Lung Dis Treat 2: 107. doi:10.4172/2472-1018.1000107

Page 9 of 11

\section{Results of sputum level of IL-8 and TNF- $\alpha$}

In seven studies with 438 participants, the sputum level of IL-8 was found to be significantly reduced $[16,18,20,31,38-40]$ (MD $-0.88,95 \%$ CI $[-1.45,-0.31])(\mathrm{p}=0.002))$ (Figure 7).

In four studies with 206 participants, the sputum level of TNF- $\alpha$ was found to be significantly reduced $[20,25,31,40]$. Due to the wide range of values of TNF- $\alpha$ among these studies, Std.MD was used in the analysis (Std.MD -1.05, 95\% CI [-1.97, -0.13]) ( $\mathrm{p}=0.02)$ (Figure 8).

\section{Adverse events}

Minor adverse events (AEs), such as abdominal distension, were found in five patients in one study [40]; two studies reported no AEs $[16,24]$; Wang [29] reported similar percentage ratio of subjects that experienced AEs. The remaining studies did not mention occurrence of AEs.

\section{Discussion}

This SR included 29 RCTs, and focused on the change in concentration levels of inflammatory mediators in both serum and sputum in patients with stable COPD. The experimental group received oral CHM (in the form of pill, tablet, granule, capsule, or decoction) plus bronchodilators (per GOLD guideline). The control group received bronchodilators, either alone or with placebo. Six studies were found in English databases and twenty three in Chinese databases. Twenty seven studies were conducted in China and two in Japan.

The methodological quality was of low risk-of-bias for all domains in two studies. The quality of evidence was assessed as low by GRADEpro for the meta-analysis of TNF- $\alpha$ in induced sputum, and moderate for other inflammatory mediators.

The study findings indicated that certain CHM formulae appear to reduce systemic inflammatory response in patients with stable COPD. A significant reduction in the concentration of IL-8, IL- 6 , and TNF- $\alpha$, and TGF- $\beta 1$ in serum, and IL- 8 and TNF- $\alpha$ in induced sputum were found in the experimental groups compared to control groups. In addition, a statistically significant higher heterogeneity rate was also found through meta-analysis, which maybe correlated to the duration of intervention, severity of COPD, various differentiation syndromes, usage of various bronchodilators, and subject population in each trial. Further sub-analysis was not conducted in this SR due to limitation of high amount of studies.

Three RCTs reported a change in the concentration level of IL-8, IL-6, MMP-9, and TNF- $\alpha$ in induced sputum for stable COPD patients treated with salmeterol/fluticasone, roflumilast, and nutritional supplementation [46-48]; improvement to lung function and quality of life were also observed in the same studies. Therefore, the reduced level of inflammatory mediators in either induced sputum or serum may have caused a decrease in airway inflammation, which presumably explains the MOA.

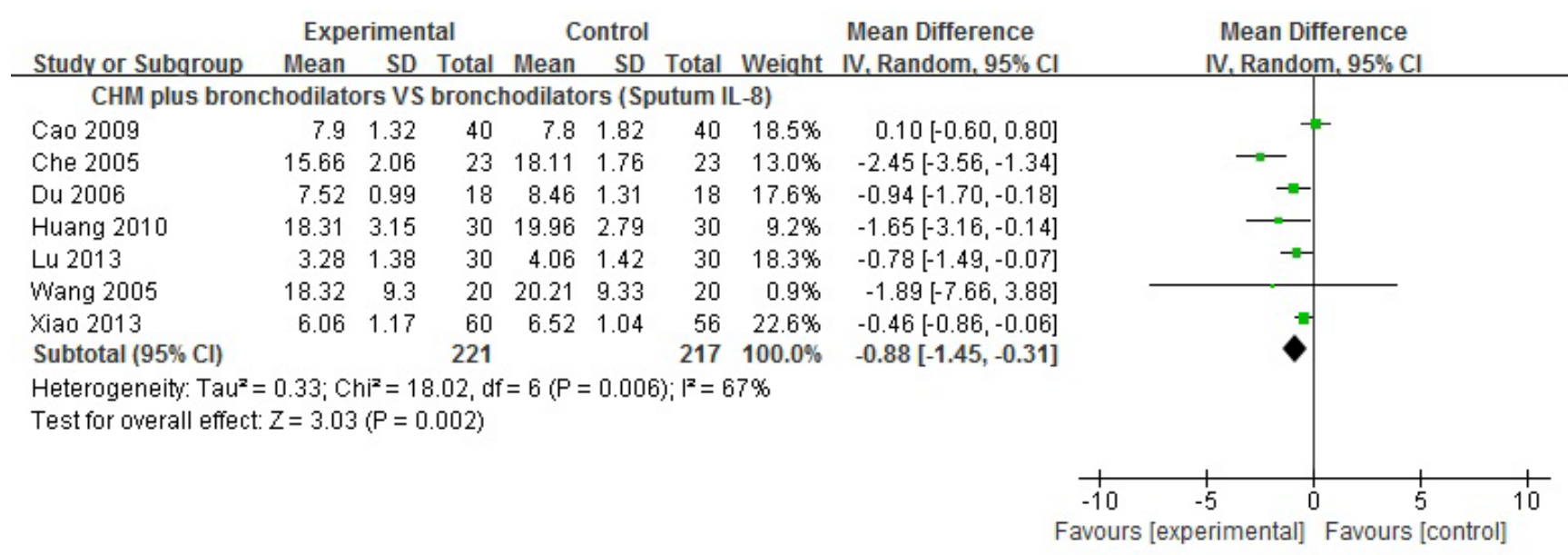

Figure 7: Meta-analysis of CHM plus bronchodilators versus bronchodilators with reduction of sputum IL-8 at the end of treatment as the outcome.

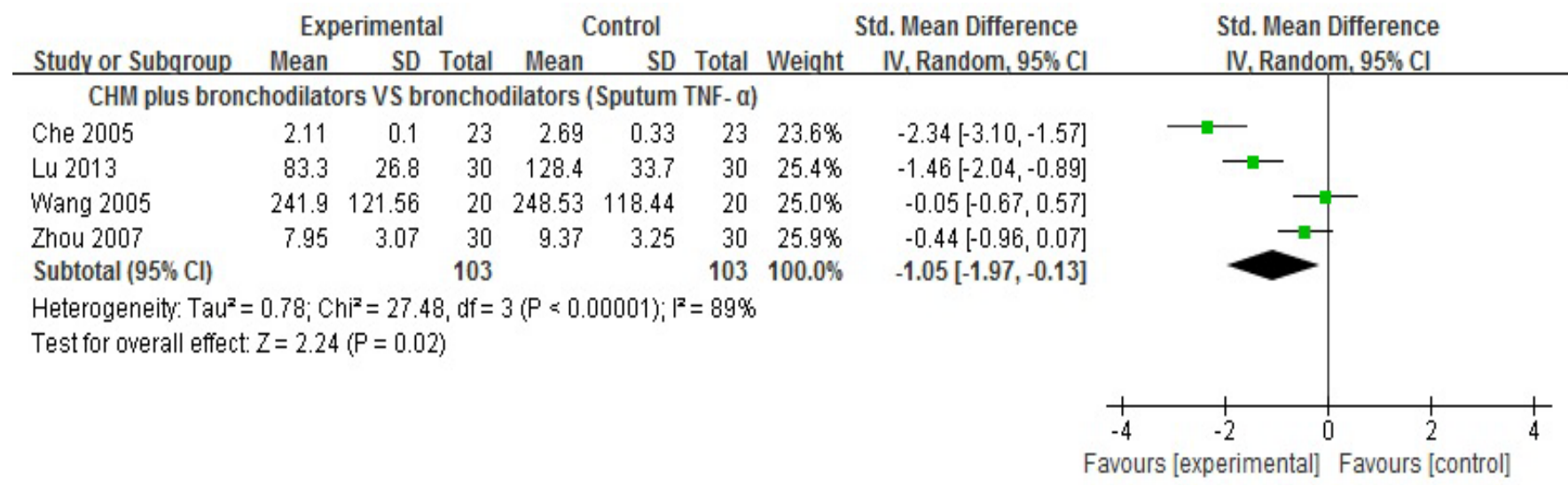

Figure 8: Meta-analysis of CHM plus bronchodilators versus bronchodilators with reduction of sputum TNF- $\alpha$ at the end of treatment as the outcome. 
TNF- $\alpha$, IL-8 in serum, and lung function were assessed in twelve studies [22-24,26,27,29,32,34-37,41]. The QoL was assessed by the St. George Respiratory Questionnaire in five studies [19,24,29,30,37]. TNF- $\alpha$ in sputum and lung function was assessed in three studies $[25,31,40]$. All results demonstrated that usage of adjunctive CHM had similar anti-inflammatory effect as salmeterol/fluticasone, roflumilast and nutritional supplementation, which further indicates CHM's potential MOA.

The theory of Chinese Medicine (CM) defines COPD as lung distension, and its differentiation of syndromes (differential diagnosis) include phlegm retention and deficiency of organs, which mainly correlates to deficiencies in lung, spleen, and kidney function. The goal of $\mathrm{CM}$ is to replenish the lung, invigorate the spleen, and tonify the kidney. In the 29 studies, the two most commonly used formulae were Bu Fei Tang (replenish lung) and Bu Zhong Yi Qi Tang (invigorate spleen). The most commonly used herbs consisted of Huang Qi (Astragalus membranaceus), Bai Zhu (Atractylodes macrocephala), Dang Shen (Codonopsis pilosula), and $\mathrm{Wu}$ Wei Zi (Schisandra chinensis).

Previous studies (on animal models) have shown that Bu Fei Tang affected the expression of MMP-9 on airway remodeling, and significantly reduced the level of TNF- $\alpha$ and IL-8 in a COPD rat model in Bronchial Alveolar Lavage Fluid with lung Qi deficiency [49,50]. Bu Zhong Yi Qi Tang has been shown to increase the rat T-lymphocytes division and the amount of IL-2 produced in mice with spleen deficiency [51].

Ginseng is consisted of ginsenosides and ginseng polysaccharides. Its pharmacological actions have been investigated worldwide. In mice, the extract was found to decrease airway inflammation [52].

Astragalus was found to modify responses of lipopolysaccharidestimulated macrophages and reduce the production of TNF- $\alpha$, IL- 6 and IL-10 [53]. Dang Shen extract (Codonopsis pilosula) was found to suppress the release of TNF- $\alpha$, also indicating anti-inflammatory effects [54].

One of the components of Wu Wei Zi (Schisandra chinensis) is Schisandrin B, which down-regulated the production of proinflammatory mediators, such as TNF- $\alpha$ and IL-6. Bai Zhu (Atractylodes macrocephala) extracts were found to have anti-inflammatory effects on TNF- $\alpha$ and nitric oxide production from peritoneal macrophages in mice [55] and in a rat lung cell membrane chromatography model [56].

Based on clinical studies and experiments, the MOA of CHM on COPD includes: 1. Decrease in cytokine levels and suppression of airway inflammation; 2. Improvement of overall immune functions; 3. Maintenance of oxidant-antioxidant balance; and 4. Regulation of proteases and anti-proteases levels [57].

However, due to inconsistent methods used to measure inflammatory mediators, the small number of studies, the small sample size, and poor quality of methodology of certain studies, the effect of CHMs on inflammatory mediators could not be completely confirmed. Moreover, AEs related to liver and kidney function should be investigated in future clinical trials. Further, RCTs on CHM therapy should be reported through CONSORT $2010[14,58]$.

\section{Conclusion}

This SR explains CHM's mechanism of action, demonstrates CHM's anti-inflammatory effects, and shows that $\mathrm{CHM}$ is well tolerated by patients with stable COPD. Furthermore, using CHM adjunctively has shown to be beneficial in treating and slowing the progression of COPD.

\section{Author's Contribution}

Dr. Xuedong An and Dr Qing Miao are the guarantor, and will take responsibility for the manuscript, including the data and analysis of data. They contributed to the concept and design of this systematic review. XC, CQ, and BW contributed to data research and extraction. Dr. Yifei Du, Xiaodong Cong and Carole Yujia Qiao had full access to all the data in the study and take responsibility for the integrity of the data, accuracy of data analysis, and interpretation of data. Xuedong An and Carole Yujia Qiao contributed to writing the first draft of this manuscript. Xiaodong Cong Bing Wang, and Qing Miao contributed by reviewing the manuscript. Xuedong and Yifei Du contributed to the final revision of the manuscript.

\section{Funding}

This study was funded by Beijing Municipal Science and Technology Commission of China (NO.Z131107002213053). The sponsors were not involved in this manuscript.

\section{Acknowledgement}

We thank the Beijing Municipal Science and Technology Commission of China for supporting this study.

\section{References}

1. Hogg JC, Timens W (2009) The pathology of chronic obstructive pulmonary disease. Annu Rev Pathol 4: 435-459.

2. Chung KF (2001) Cytokines in chronic obstructive pulmonary disease. Eur Respir J Suppl 34: 50s-59s.

3. Hacievliyagil SS, Mutlu LC, Temel I (2013) Airway inflammatory markers in chronic obstructive pulmonary disease patients and healthy smokers. Niger $J$ Clin Pract 16: 76-81.

4. Petrescu F, Voican SC, Silosi I (2010) Tumor necrosis factor-alpha serum levels in healthy smokers and nonsmokers. Int $\mathrm{J}$ Chron Obstruct Pulmon Dis 5: 217-222.

5. Hacievliyagil SS, Gunen H, Mutlu LC, Karabulut AB, Temel I (2006) Association between cytokines in induced sputum and severity of chronic obstructive pulmonary disease. Respir Med 100: 846-854.

6. Gaki E, Kontogianni K, Papaioannou Al, Bakakos P, Gourgoulianis KI, et al. (2011) Associations between BODE index and systemic inflammatory biomarkers in COPD. COPD 8: 408-413.

7. Gan WQ, Man SF, Senthilselvan A, Sin DD (2004) Association between chronic obstructive pulmonary disease and systemic inflammation: a systematic review and a meta-analysis. Thorax 59: 574-580.

8. An X, Zhang AL, May BH, Lin L, Xu Y, et al. (2012) Oral Chinese herbal medicine for improvement of quality of life in patients with stable chronic obstructive pulmonary disease: a systematic review. J Altern Complement Med 18: $731-743$

9. Guo R, Pittler MH, Ernst E (2006) Herbal medicines for the treatment of COPD: a systematic review. Eur Respir J 28: 330-338.

10. Eden J, Levit L, Berg A, Morton S (2011) Finding What Works in Health Care: Standards for Initiating a Systematic Review, Chapter 2, P74. Washington (DC) National Academies Press (US).

11. Moher D, Liberati A, Tetzlaff J, Altman DG (2010) Preferred reporting items for systematic reviews and meta-analyses: the PRISMA statement. Int J Surg 8 : 336-341.

12. Hand book (2016) Cochrane Handbook for Systematic Reviews of Interventions Part I, Chapter 8 (Version 5.0.2).

13. Guyatt G, Oxman AD, Akl EA, Kunz R, Vist G, et al. (2011) GRADE guidelines: 1. Introduction-GRADE evidence profiles and summary of findings tables. J Clin Epidemiol 64: 383-394.

14. Gagnier JJ, Boon H, Rochon P, Moher D, Barnes J, et al. (2006) Reporting randomized, controlled trials of herbal interventions: an elaborated CONSORT statement. Ann Intern Med 144: 364-367.

15. CSRD (2007) Chinese Society of Respiratory Disease (CSRD): Guideline for diagnosis and management of chronic obstructive pulmonary disease. Chin Inrern Med 46: 254-261.

16. Du C, Tu M, Liu W, Liu Y, Liu X (2006) Effects of Ginkgo Biloba extact (GBE) on the inflammatory cell and the level of interlukin-8 in induced sputum from patients with chronic obstructive pulmonary disease. The Journal of Practical Medicine 22: 1246-1248.

17. Guo S, Sun Z, Liu E, Feng J, Fu M, et al. (2014) Effect of bufei granule on stable chronic obstructive pulmonary disease: a randomized, double blinded, placebocontrolled, and multicenter clinical study. J Tradit Chin Med 34: 437-444.

18. Huang B (2010) Effect of Chinese herbal medicine of Bufei Yiyang Huatan on cytokine cells IL-10 $\square \mathrm{IL}-8$ in stable chronic obstructive pulmonary disease patients. Chinese Medicine Modern Distance Education of China 8: 144-145. 
19. Li Z, Tian C, Liu W (2013) Clinical study of Yiqijianpi recipe for treating stable chronic obstructive pulmonary disease. Acta Chinese Medicine and Pharmacology 41: 119-120.

20. Lu F, Wang S (2013) Influence of Liujunzi Decoction on Endogenous Antiinflammatory Mechanisms in Patients with Chronic Obstructive Pulmonary Disease. Journal of Liaoning University of TCM 7: 161-163.

21. Zhong M, Ye X, Li D (2012) Effect of Jiawei Jinshuiliujunjian on cytokine in patients with stable chronic obstructive pulmonary disease. Chinese Journal of Geriatric Care 10: 11-12.

22. Hu C (2009) Integrated Bu Fei Tang and western medicine in treatment 35 cases with stable chronic obstructive pulmonary disease. Journal of Traditional Chinese Medicinal Research 22: 24-26.

23. Su H, Wu W, Feng C, Zhou S, Tian X, et al. (2005) Effects of the therapy for nourishing qi, activating circulation and dispersing phlegm on expressions of serum TGF- $\beta 1$ and IL- 8 in the patients with chronic obstructive pulmonary disease. Journal of Beijing University of Traditional Chinese Medicine 28: 48-51.

24. Tatsumi K, Shinozuka N, Nakayama K, Sekiya N, Kuriyama T, etal. (2009) Hochuekkito improves systemic inflammation and nutritional status in elderly patients with chronic obstructive pulmonary disease. J Am Geriatr Soc 57: 169-170.

25. Zhou Y, Wei G, Qi S, Qi Y, Shi Y, et al. (2007) Clinical study on the effects of treating stable chronic obstructive pulmonary disease with Fei Sai Tong $\mathrm{He} \mathrm{Ji}$. Journal of Taishan medical college 28: 515-517.

26. Shinozuka N, Tatsumi K, Nakamura A, Terada J, Kuriyama T (2007) The traditiona herbal medicine Hochuekkito improves systemic inflammation in patients with chronic obstructive pulmonary disease. J Am Geriatr Soc 55: 313-314.

27. Xiao H, Chen R, Zou M, Xiong C, Zhang J (2014) Regulatory Effect of Zhenq Fuzheng Granule on Th1/Th2 in Lung-kidney-yin Deficiency Type of Stable COPD Patients. Chinese and foreign medical research 12: 8-10.

28. Wang Q, Wang L, Xu L, Huang S, Wu Z (2011) Curative effects of compund Yifei Duqi capsules on stable chronic obstructive pulmonary disease and effect of it on serum interleukin-8. Journal of TCM Univ of Hunan 31: 58-60.

29. Wang G, Liu B, Cao Y, Du Y, Zhang H, et al. (2014) Effects of two Chinese herbal formulae for the treatment of moderate to severe stable chronic obstructive pulmonary disease: a multicenter, double-blind, randomized controlled trial. PLoS One 9: e103168.

30. Feng C, Wu W, Wu H, Su H, Zhou G, et al. (2007) Analysis on clinical materials from 76 cases of COPD treated with qi-replenishing, blood-activating and phlegm-removing therapy. Journal of Beijing University of Traditional Chinese Medicine 30: 419-422.

31. Wang S, Ji H, Zhang N, Zhuo X, Zhao L, et al. (2005) Effect of Yifei Jianpi Recipe on Inflammatory Cells, Levels of Interleukin-8 and Tumor Necrosis Factor- $\alpha$ in Sputum from Patients with Chronic Obstructive Pulmonary Disease. CJITWM 25: 111-113.

32. OU J, Liu L (2013) Chronic Obstructive Pulmonary Disease in Stable Stage Treated with Jiajian Bufei Decoction. Chinese Journal of Experimental Traditional Medical Formulae 19: 303-306.

33. Zhang H, Cao F, Wang M, Li S, Ma L, et al. (2013) Influence of systemic inflammatory response and human T-cell subset numbers in stable chronic obstructive pulmonary disease by differentiation treatment with traditional Chinese medicine. Chinese Journal of Gerontology 33: 5225-5228.

34. Xiong G, Chen S, Xie W, Ye X, Lin J (2008) Clinical observation of Shenge granule on effect of lung function and serum IL-8, TNF-a for stable chronic obstructive pulmonary disease. Chinese Journal of Geriatric Care 6: 37-39.

35. Zhao T, Xu H (2012) Effect of Bailing Capsule on Lung function and serum IL-8 in patients with stable chronic obstructive pulmoary disease. Chinese Journal of Clinical Research 25: 665-666.

36. Li S, Fan L, Guo S, He Q (2013) A study of the mechanism of Jianpi Huoxue therapy in treatment of stable chronic obstructive pulmonary disease by improving airway inflammation. Modern Journal of Integrated Traditional Chinese and Western Medicine 22: 2398-2400.

37. Chen X, Ke J, Liu Q, Sheng L (2014) Discussion About Bufei Huoxue Decoction Combined Salmeterol Fluticasone Treatment on Chronic Obstructive Pulmonary Disease in Curative Effect and Action Mechanism. Chinese Journal of Experimental Traditional Medical Formulae 20: 220-223.

38. Cao D (2009) Effects of Xiaoqilongtang decoction on airway inflammation and airway remodeling in patients with COPD. Med J West China 21: 575-577.
39. Xiao B, Liu H, Zeng Y, Lao J, Chen K (2013) Effect of Manzhi Kechuanling on Inflammatory Cells and MMP-9-IL-8-CRP levels in Sputum of Chronic Obstructive Pulmonary Disease Patients. Journal of new Chinese medicine 45: 34-36.

40. Che H, Pang J, Changjian L, Wang Y (2005) Effects of Zhike Qingfei Oral Liquid on Inflammatory Cell Interleulin-8 and Tumor Necrosis Factor- $\alpha$ in the Sputum in 25 Cases of Chronic Obstructive Pulmonary Disease. Journal of Traditional Chinese Medicine 16: 759-761.

41. Cheng L, Liu X (2013) Influence of Suhuang Zhike Capsule Combined with Seretide on Pulmonary function and Proinflammatory Factors in Elderly Chronic Obstructive Pulmonary Disease. China Pharmaceuticals 22: 16-17.

42. Fu M, Sun Z, Liu E, Feng J, Wang Q (2012) Impacts of Bufei Granules on Serum IL-6 level for COPD Patients at the Stable Stage. Would Journal of Integrated Traditional and Western Medicine 7: 529-531.

43. Zheng Y (2010) Influence of traditional Chinese medicine Jinshuibao capsule on levels of serum cytokines in patients with chronic obstructive pulmonary disease. China Journal of Modern Medicine 20: 1096-1098.

44. Jiang J, Li F (2014) The influence on Lung Function and Inflammatory Mediators of Bufeinashen Decoction in Patients with Stable Chronic Obstructive Pulmonary Disease. Practical Clinical Journal of Integrated Traditional Chinese and Western Medicine 14: 1-3.

45. Vestbo J, Hurd SS, Agusti AG, Jones PW, Vogelmeier C, et al. (2013) Globa strategy for the diagnosis, management, and prevention of chronic obstructive pulmonary disease: GOLD executive summary. Am J Respir Crit Care Med 187: 347-365

46. Sugawara K, Takahashi H, Kashiwagura T, Yamada K, Yanagida S, et al. (2012) Effect of anti-inflammatory supplementation with whey peptide and exercise therapy in patients with COPD. Respir Med 106: 1526-1534.

47. Perng DW, Tao CW, Su KC, Tsai CC, Liu LY, et al. (2009) Anti-inflammatory effects of salmeterol/fluticasone, tiotropium/fluticasone or tiotropium in COPD. Eur Respir J 33: 778-784.

48. Grootendorst DC, Gauw SA, Verhoosel RM, Sterk PJ, Hospers JJ, et al (2007) Bredenbroker D, Bethke TD, Hiemstra PS, Rabe KF: Reduction in sputum neutrophil and eosinophil numbers by the PDE4 inhibitor roflumilast in patients with COPD. Thorax 62: 1081-1087.

49. Zhang K, Liu LL, OU JQ, Zhan HJ, Liu HR (2008) Effect of Jia Jian Bu Fei decoction on TNF-alpha and IL-8 of bronchoalveolar lavage fluid in COPD rats with deficiency of lung-qi. Tianjin Journal of Traditional Chinese Medicine 25 491-493.

50. Zhang K, Zhang Y, Cheng YJ, Lu L (2008) Effects of Shenqi Bufei Tang on expessions of NF-kappaB, MMP-9 and TIMP-1 in airway remodeling of COPD rat model with lung-Qi deficiency syndrome. Zhongguo Zhong Yao Za Zhi 33: 2129-2132.

51. Hu B, An HM, Shen KP (2008) Pharmaceutical study on Bu Zhong Yi Qi Tang for anti-infection, anti-tumor and immuno function. Central south pharmacy 6 : 731-734.

52. Kim DY, Yang WM (2011) Panax ginseng ameliorates airway inflammation in an ovalbumin-sensitized mouse allergic asthma model. J Ethnopharmacol 136 230-235.

53. Clement-Kruzel S, Hwang SA, Kruzel MC, Dasgupta A, Actor JK (2008) Immune modulation of macrophage pro-inflammatory response by goldensea and Astragalus extracts. J Med Food 11: 493-498.

54. Byeon SE, Choi WS, Hong EK, Lee J, Rhee MH, et al. (2009) Inhibitory effect of saponin fraction from Codonopsis lanceolata on immune cell-mediated inflammatory responses. Arch Pharm Res 32: 813-822.

55. Li CQ, He LC, Jin JQ (2007) Atractylenolide I and atractylenolide III inhibit Lipopolysaccharide-induced TNF-alpha and NO production in macrophages. Phytother Res 21: 347-353.

56. Dong H, He L, Huang M, Dong Y (2008) Anti-inflammatory components isolated from Atractylodes macrocephala Koidz. Nat Prod Res 22: 1418-1427.

57. Le $Y$, Sun $Y$ (2013) The progress of Chinese medicine for prevention and treatment of chronic obstructive pulmonary disease mechanism. Global Traditional Chinese Medicine 6: 226-229.

58. Schulz KF, Altman DG, Moher D (2010) CONSORT 2010 statement: updated guidelines for reporting parallel group randomized trials. Ann Intern Med 152 726-732. 